\title{
Tunneling in an anisotropic cubic Dirac semi-metal
}

\author{
Ahmed Bouhlal $^{\mathrm{a}}$, Ahmed Jellal ${ }^{\mathrm{a}}$, Hocine Bahlouli $^{\mathrm{b}}$, Michael Vogl $^{\mathrm{b}}$ \\ ${ }^{a}$ Laboratory of Theoretical Physics, Faculty of Sciences, Chouaïb Doukkali University, 24000 El Jadida, Morocco \\ ${ }^{b}$ Department of Physics, King Fahd University of Petroleum and Minerals, 31261 Dhahran, Saudi Arabia
}

\begin{abstract}
Motivated by a recent first principles prediction of an anisotropic cubic Dirac semi-metal in a real material $\mathrm{Tl}(\mathrm{TeMo})_{3}$, we study the behavior of electrons tunneling through a potential barrier in such systems. To clearly investigate effects from different contributions to the Hamiltonian we study the model in various limits. First, in the limit of a very thin film material where the linearly dispersive $z$-direction is frozen out at zero momentum and the dispersion in the $x-y$ plane is rotationally symmetric. In this limit we find a Klein tunneling reminiscent of what is observed in single layer graphene and linearly dispersive Dirac semi-metals. Second, an increase in thickness of the material leads to the possibility of a non-zero momentum eigenvalue $k_{z}$ that acts as an effective mass term in the Hamiltonian. We find that these lead to a suppression of Klein tunneling. Third, the inclusion of an anisotropy parameter $\lambda \neq 1$ leads to a breaking of rotational invariance. Furthermore, we observed that for different values of incident angle $\theta$ and anisotropy parameter $\lambda$ the Hamiltonian supports different numbers of modes propagating to infinity. We display this effect in form of a diagram that is similar to a phase diagram of a distant detector. Fourth, we consider coexistence of both anisotropy and non-zero $k_{z}$ but do not find any effect that is unique to the interplay between non-zero momentum $k_{z}$ and anisotropy parameter $\lambda$. Last, we studied the case of a barrier that was placed in the linearly dispersive direction and found Klein tunneling $T-1 \propto \theta^{6}+\mathcal{O}\left(\theta^{8}\right)$ that is enhanced when compared to the Klein tunneling in linear Dirac semi-metals or graphene where $T-1 \propto \theta^{2}+\mathcal{O}\left(\theta^{4}\right)$.
\end{abstract}

\section{Contents}

1 Introduction

2 Theoretical model $\quad 2$

3 Analysis of different limits 3

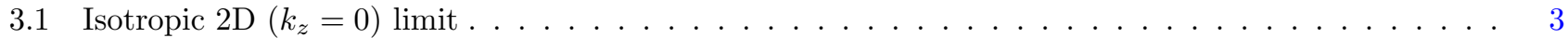

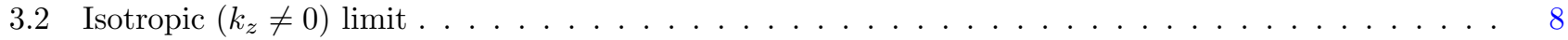

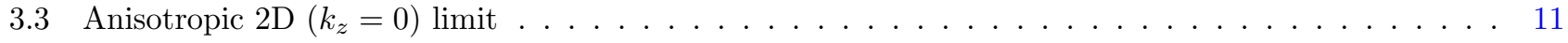

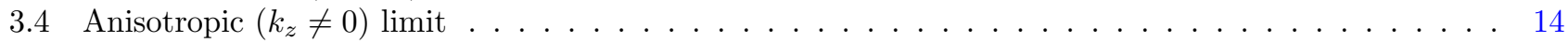

3.5 Bulk transmission through a potential along z-direction $\ldots \ldots \ldots \ldots \ldots \ldots$

4 Conclusion $\quad 18$

5 Acknowledgements $\quad 19$

$\begin{array}{llll}\text { Appendix } & \text { A } & \text { The 2D limit - freezing out } k_{z} & 28\end{array}$

$\begin{array}{ll}\text { Appendix B Wavefunctions in the different spatial regions } & 29\end{array}$

Appendix B.1 Wavefunctions for the isotropic $\left(k_{z} \neq 0\right)$ limit . . . . . . . . . . . . . . . 29

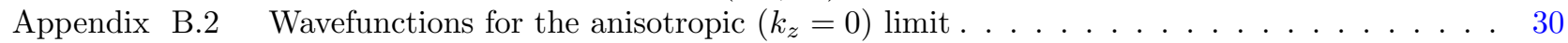

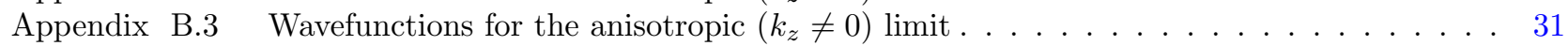

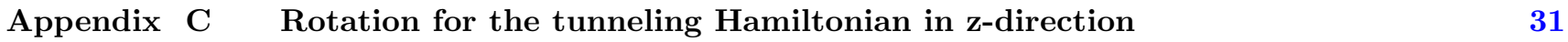

Email address: ssss133@googlemail.com (Michael Vogl) 


\section{Introduction}

After the discovery of graphene [1] - a two-dimensional lattice of carbon atoms that are arranged in a honeycomb pattern forming a hexagonal lattice - two dimensional materials have become a hot topic both experimentally and theoretically. After all, graphene hosts overwhelmingly many exotic and intriguing phenomena [2-35]. This and the many promising and fascinating physical, electrical, chemical, magnetic and optical properties[36-44] have since motivated considerable efforts that were deployed to study properties of other novel 2D materials. Examples include similar thin materials such as silicene[45-49], transition metal dichalcogenides[50-58] and very importantly a large amount of work on multi-layer materials[59-94] to name a few.

However, it was soon realized that the peculiar properties of graphene are not just a consequence of its low dimensionality but rather due to its linear band structure. This naturally led to a search for similar materials in higher dimensions $[95,96]$ and other generalizations that all are now characterized as Dirac and Weyl semi-metals.

Weyl semi-metals [96-102, 102, 103, 103-127] and Dirac semi-metals [95-97, 108, 109, 117, 128-150] are considered to be the 3D analogues of graphene materials. The common feature that is shared by linear 3D Dirac and Weyl semi-metals is the fact that conduction and valence bands touch each other at single, discrete points in $k$-space, around which the dispersion has a linear $k$-dependence. This unusual band dispersion causes the electrons around the Fermi energy to behave like relativistic particles similarly to graphene systems. However, band touching points need not necessarily be linear and higher order band-crossings are possible [98, 118, 127, 130, 148]. Even more generally, band crossings in Dirac and Weyl semimetals (called "Dirac" or "Weyl" points) can even have different order of dispersion in different directions as dictated by their crystal symmetries. It has been realized recently that these Dirac and Weyl band-crossing points carry important information on the topological behavior of the system, giving rise to specific behaviors of surface or edge states in such systems [1, 98, 151]. These gapless degrees of freedom are protected by the topological properties of the system and define the so-called topological invariants of the system. Thus Dirac and Weyl semimetals possess non-trivial protected topological properties that are at the origin of some of their exciting electronic and transport properties. Of course there exists a large zoo of similar materials that might not just have band touching points but where bands might even touch along nodal lines or on surfaces [152-171]. Many semi-metals have been studied in some detail including their tunneling properties but not all of them. Particularly, not all semi-metals with higher order band crossing points have been studied exhaustively. Some recent examples that have been studied include multi Weyl semi-metals with a dispersion that is rotationally symmetric in the $\mathrm{x}-\mathrm{y}$ plane [172-174] and higher psuedo-spin generalizations of Weyl semi-metals [175].

In our present work we study the behavior of electrons tunneling through a potential barrier in an "anisotropic cubic Dirac fermion" system like the one that was recently predicted based on first principles computations for $\mathrm{Tl}(\mathrm{TeMo})_{3}$ and related materials, which are real and stable solid state systems [98]. It should be stressed that we study a dispersion that is also anisotropic in the $\mathrm{x}-\mathrm{y}$ plane but keeps a sixfold rotational symmetry.

The work is organized as follows. In section 2 we introduce the mathematical model we consider, in section 3.1 we study the $2 \mathrm{D}$ limit of a thin material that has momentum in $z$-direction frozen out at zero value (setting $\left.k_{z}=0\right)$ and therefore behaves approximately as a two-dimensional system. Here we find Klein tunneling behaviour that is very reminiscent of what is known in graphene or linearly dispersive semi metals. In section 3.2, we consider the case of a cubic Dirac semi metal that is isotropic in the plane but allows a finite momentum $k_{z}$ in $z$-direction. The momentum term acts similarly to a mass term and therefore spoils some of the Klein tunneling properties. In section 3.3 we consider the anisotropic case with $k_{z}=0$, where we discover that for different angles and anisotropy strengths tunneling properties can differ wildly. In section 3.4 we consider the anisotropic case but with a non zero $k_{z}$ value and the addition of the mass-term mainly serves to damp tunneling phenomena. Lastly, in section 3.5 we consider tunneling through a potential barrier along the linearly dispersive $z$-direction in a bulk cubic Dirac semi-metal, where we discover Klein tunneling that is enhanced compared to the Klein tunneling that is known from graphene and linearly dispersive semi-metals. Finally, in 4 we present our conclusion concerning our main findings and discuss some open questions.

\section{Theoretical model}

The Hamiltonian describing an anisotropic cubic Dirac semi-metal can be written as follows [98]

$$
H(k)=\hbar\left(\begin{array}{cc}
h(k) & 0 \\
0 & -h(k)
\end{array}\right)+V(x) \rrbracket_{4}
$$

where

$$
h(k)=v_{x} \sigma_{x}\left(\hat{k}_{+}^{3}+\hat{k}_{-}^{3}\right)+i v_{y} \sigma_{y}\left(\hat{k}_{+}^{3}-\hat{k}_{-}^{3}\right)+v_{z} k_{z} \sigma_{z}
$$


An isotropic version of this model was also presented in [148] and the terms also appears in the context of spin-orbit coupling corrections [176]. In the equation, we have $\hat{k}_{i}$ as momentum operators. We used the shorthand notation $\hat{k}_{ \pm}=\hat{k}_{x} \pm i \hat{k}_{y}, \sigma_{i}$ are Pauli matrices and $v_{x, y, z}$ are independent real coefficients with dimension $T^{-1}$ ( $\mathrm{T}$ stands for time). That is, wavevector $\mathbf{k}$ and positions $\mathbf{x}$ are chosen dimensionless. Typically in the real solid state system that realizes the above Hamiltonian it is written in a basis set that corresponds to a wavefunction of the form $|\psi\rangle=(|A, \uparrow\rangle,|B, \uparrow\rangle,|A, \downarrow\rangle,|B, \downarrow\rangle)^{T}$, where $A$ and $B$ denote some orbital degrees of freedom and $\uparrow / \downarrow$ spin degrees of freedom [98].

We consider the case where the system is subject to a scalar potential barrier of the form

$$
V(x)=\left\{\begin{array}{ll}
V_{0}, & -\frac{L}{2}<x<\frac{L}{2} \\
0, & \text { elsewhere }
\end{array},\right.
$$

which is also shown in Fig. 1:

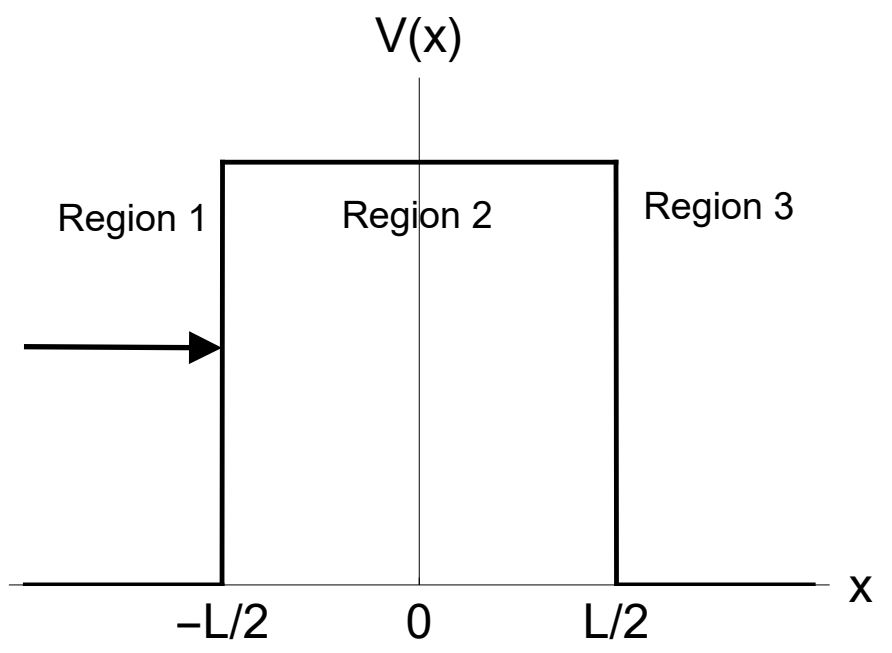

Figure 1: The figure schematically shows the setting of our scattering problem and introduces the labeling scheme for the different potential regions.

This type of Hamiltonian has several interesting properties, which are the cubic dispersion, anisotropy in the $x$ - $y$ plane and linear momentum dependence in $z$-direction. To fully understand the effect of all these contributions to the tunneling phenomena we will study the effects of each of these contributions separately. That is, we will first study simplest case of the rotationally symmetric 2D limit $v_{x}=v_{y}$ with $k_{z}=0$. Afterwards we will investigate the effects of non-zero $k_{z}$, then the anisotropic limit $v_{x} \neq v_{y}$ with $k_{z}=0$ and finally the case of both $k_{z} \neq 0$ and $v_{x} \neq v_{y}$.

\section{Analysis of different limits}

\subsection{Isotropic $2 D\left(k_{z}=0\right)$ limit}

As a first step and to gain some intuition about the problem at hand, we consider the $2 D$ flat limit by setting $k_{z}=0$. Furthermore, we consider the special case of an isotropic dispersion setting $v_{x}=v_{y}=v$ for simplicity. The limit of $k_{z}=0$ is relevant because typically $v_{z} \gg v_{x, y}$ and therefore $k_{z}$ is typically frozen out if the sample is sufficiently thin (see appendix Appendix A for details).

Under these simplifying conditions one may write the Hamiltonian of the system in a matrix form

$$
H(k)=\left(\begin{array}{cccc}
V(x) & 2 \hbar v \hat{k}_{+}^{3} & 0 & 0 \\
2 \hbar v \hat{k}_{-}^{3} & V(x) & 0 & 0 \\
0 & 0 & V(x) & -2 \hbar v \hat{k}_{+}^{3} \\
0 & 0 & -2 \hbar v \hat{k}_{-}^{3} & V(x)
\end{array}\right) .
$$

In order to solve this eigenvalue problem we can separate variables and write the eigenspinors as plane waves in the $y$-direction. This is due to the fact that $\left[H, k_{y}\right]=0$ implies conservation of momentum along the $y$-direction $\Psi(x, y)=e^{i k_{y} y} \psi(x)$. 
Solutions in each region are then given by plane waves $\psi(x) \propto e^{ \pm i k_{x} x}$. It is convenient to switch to dimensionless variables by defining the reduced energy $\epsilon$ and potential $u$

$$
\epsilon=\frac{E}{2 \hbar v} ; \quad u_{j}=\frac{V_{j}}{2 \hbar v}
$$

where $V_{j}$ is the potential in region $j$ (compare Fig. 1). The reduced energy eigenvalues in each region therefore are given by

$$
\epsilon_{j}= \pm\left(k_{x}^{2}+k_{y}^{2}\right)^{\frac{3}{2}}+u_{j} .
$$

Since this reduced energy formula is rotationally symmetric the result can be plotted as a function of the wavevector amplitude $k=\left(k_{x}^{2}+k_{y}^{2}\right)^{\frac{1}{2}}$, which is shown in the Fig. 2.
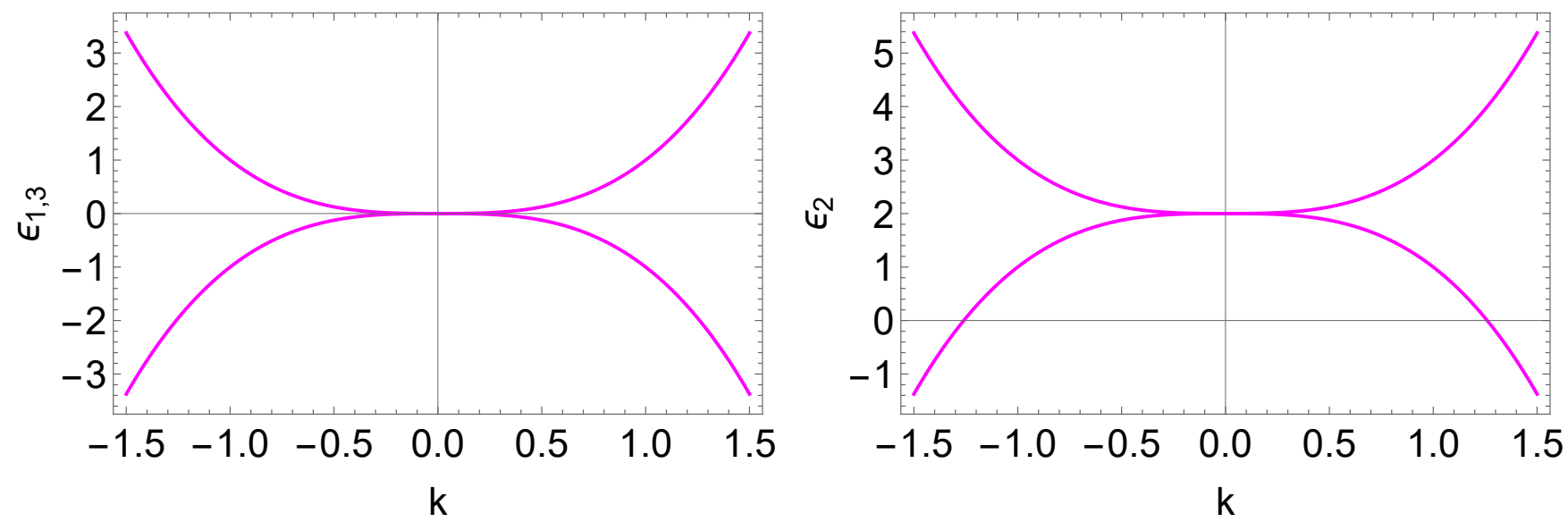

Figure 2: (color online) Reduced energy $\epsilon$ in different regions as a function of the incident wavevector amplitude $k$. The left panel shows regions 1 and 3 and the right panel shows region 2 .

We find that the band touching point is very flat which means that kinetic energy of carriers is very small in these regions and hence interactions dominate these regions.

\subsubsection{Solution of the tunneling problem}

We now turn to the tunneling problem at a fixed value of $\epsilon$. The energy $\epsilon$ is determined by the wavevector $\mathbf{k}=(\cos \theta, \sin \theta) k$ of the incoming wave. It is therefore convenient to recast the problem as a generalized cubic eigenvalue problem for $k_{x}$ rather than a linear energy eigenvalue problem for $\epsilon$. This is because the problem is more clearly understood in this language.

After all, for the tunneling problem at hand one needs to match wavefunctions, their derivatives and second derivatives to ensure smoothness because the Hamiltonian includes up to third order derivatives. At each boundary region there are four matching conditions (one for each vector component). The same is true for the matching conditions of first and second derivatives. This means that at each potential jump one ends up with a total of 12 matching conditions. A linear combination of four eigenvectors in each region as an eigenenergy problem naively suggests, would not provide enough free constants. However, for a generalized cubic non-linear eigenvalue problem for $k_{x}$ one can find 12 solutions in each region (with only some of them contributing), which is sufficient to fulfill the boundary value problem.

The generalized cubic eigenvalue problem for $k_{x}$ therefore reads

$$
\left[k_{x}^{3} \tau_{z} \otimes \sigma_{x}+3 i k_{y} k_{x}^{2} \tau_{z} \otimes \sigma_{2}-3 k_{y} k_{x} \tau_{z} \otimes \sigma_{1}-i k_{y}^{3} \tau_{z} \otimes \sigma_{y}+\left(u_{j}-\epsilon\right) \mathbb{1}_{2} \otimes \mathbb{1}_{2}\right] \psi=0 .
$$

It is customary for $2 \mathrm{D}$ tunneling problems to choose $k_{y}=k \sin \theta$ to use the notion of incident angle and express the energy in terms of wavevector amplitude $k$ as $\epsilon_{j}= \pm k^{3}-u_{j}$. Doing so, one finds the following six $k_{x}$ eigenvalues that are each two-fold degenerate

$$
\begin{aligned}
& k_{x, 1}^{+}=-k_{x, 1}^{-}=k \cos (\theta) \\
& k_{x, 2}^{+}=-k_{x, 2}^{-}=-\frac{k}{\sqrt{2}}|f(\theta)|^{1 / 2} e^{i \gamma(\theta)} \\
& k_{x, 3}^{+}=-k_{x, 3}^{-}=-\frac{k}{\sqrt{2}}|f(\theta)|^{1 / 2} e^{-i \gamma(\theta)},
\end{aligned}
$$


where $\gamma(\theta)=\frac{1}{2} \arg [f(\theta)]$ and $f(\theta)=\cos (2 \theta)+i \sqrt{3}-2$, with $\sin [\gamma(\theta)]>0$. The wavefunctions therefore can be categorized as some of them being proportional to propagating plane waves $e^{ \pm i k_{x, 1}^{+} x}$ while others $e^{ \pm i k_{x,(2,3)}^{+} x}$ have exponentially growing or decaying amplitudes.

To build the solution of our problem we need to determine which of the $k_{x, i}^{ \pm}$are compatible with the boundary conditions. For simplicity, we only consider positive energies, $\epsilon>0$.

In region $1\left(x<-\frac{L}{2}\right)$ we need to have finite $\psi$ as $x \rightarrow-\infty$. We realize that $e^{i k_{x, 1}^{ \pm} x}$ is a propagating wave and therefore is allowed. Next we see that $\left|e^{i k_{x, 2}^{-} x}\right| \rightarrow \infty$ and $\left|e^{i k_{x, 3}^{+} x}\right| \rightarrow \infty$ as $x$ tends to $-\infty$ so this state is not allowed physically. Lastly, we see that $e^{i k_{x, 3}^{-} x}$ and $e^{i k_{x, 2}^{+} x}$ correspond to solutions that shrink exponentially as $x$ tends to $-\infty$ and hence are physically allowed. Therefore we have 4 physically allowed $k_{x}$ eigenvalues in this region, which corresponds to 8 wavefunctions. A linear combination of all these 8 contributions can be used as an appropriate ansatz such that

$$
\begin{aligned}
\Phi_{1}(x) & =a_{1} e_{k, 1}^{+}\left(\begin{array}{c}
\chi_{k, 1}^{+} \\
1 \\
0 \\
0
\end{array}\right)+a_{2} e_{1, k}^{+}\left(\begin{array}{c}
0 \\
0 \\
-\chi_{k, 1}^{+} \\
1
\end{array}\right)+a_{3} e_{k, 2}^{+}\left(\begin{array}{c}
-\chi_{k, 2}^{+} \\
1 \\
0 \\
0
\end{array}\right)+a_{4} e_{k, 2}^{+}\left(\begin{array}{c}
0 \\
0 \\
\chi_{k, 2}^{+} \\
1
\end{array}\right)+a_{5} e_{k, 1}^{-}\left(\begin{array}{c}
\chi_{k, 1}^{-} \\
1 \\
0 \\
0
\end{array}\right) \\
& +a_{6} e_{k, 1}^{-}\left(\begin{array}{c}
0 \\
0 \\
-\chi_{k, 1}^{-} \\
1
\end{array}\right)+a_{7} e_{k, 3}^{-}\left(\begin{array}{c}
-\chi_{k, 3}^{-} \\
1 \\
0 \\
0
\end{array}\right)+a_{8} e_{k, 3}^{-}\left(\begin{array}{c}
0 \\
0 \\
\chi_{k, 3}^{-} \\
1
\end{array}\right),
\end{aligned}
$$

where we have used the shorthand notations

$$
\chi_{k, n}^{ \pm}=\frac{\left(k_{x, n}^{ \pm}{ }^{2}+k^{2} \sin ^{2} \theta\right)^{\frac{3}{2}}}{\left(k_{x, n}^{ \pm}-i k \sin \theta\right)^{3}} ; \quad e_{k, n}^{ \pm}=e^{i k_{x, n}^{ \pm} x} .
$$

In region $2\left(-\frac{L}{2}<x<\frac{L}{2}\right)$ the presence of the potential $u$ leads to a change in wavevector $\mathbf{k}$. To have a notation that distinguishes it from the other two regions that have the same wavevector we define $q=(u-\epsilon)^{1 / 3}$ as the wavevector amplitude in region 2 and $q_{x, y}$ as its components. We now want to express everything in terms of the new wavevector amplitude $q$. Momentum conservation in $y$-direction requires that $q_{y}=k_{y}=q \sin \phi$ with $\phi=\sin ^{-1}\left(\frac{k}{q} \sin \theta\right)$. Similarly to what happened in other regions, one finds multiple solutions for $q_{x}$, which are given by Eq. (8) if we replace $k \rightarrow q$ and $\theta \rightarrow \phi$. Since region 2 is of finite size there are no restrictions on the choice of $q_{x, n}^{ \pm}$. Therefore an ansatz for the wavefunction has to include all 12 possible terms

$$
\begin{gathered}
\Phi_{2}(x)=b_{1} e_{q, 1}^{+}\left(\begin{array}{c}
-\chi_{q, 1}^{+} \\
1 \\
0 \\
0
\end{array}\right)+b_{2} e_{q, 1}^{+}\left(\begin{array}{c}
0 \\
0 \\
\chi_{q, 1}^{+} \\
1
\end{array}\right)+b_{3} e_{q, 2}^{+}\left(\begin{array}{c}
\chi_{q, 2}^{+} \\
1 \\
0 \\
0
\end{array}\right)+b_{4} e_{q, 2}^{+}\left(\begin{array}{c}
0 \\
0 \\
-\chi_{q, 2}^{+} \\
1
\end{array}\right)+b_{5} e_{q, 3}^{+}\left(\begin{array}{c}
\chi_{q, 3}^{+} \\
1 \\
0 \\
0
\end{array}\right)+b_{6} e_{q, 3}^{+}\left(\begin{array}{c}
0 \\
0 \\
-\chi_{q, 3}^{+} \\
1
\end{array}\right) \\
+b_{7} e_{q, 1}^{-}\left(\begin{array}{c}
-\chi_{q, 1}^{-} \\
1 \\
0 \\
0
\end{array}\right)+b_{8} e_{q, 1}^{-}\left(\begin{array}{c}
0 \\
0 \\
\chi_{q, 1}^{-} \\
1
\end{array}\right)+b_{9} e_{q, 2}^{-}\left(\begin{array}{c}
\chi_{q, 2}^{-} \\
1 \\
0 \\
0
\end{array}\right)+b_{10} e_{q, 2}^{-}\left(\begin{array}{c}
0 \\
0 \\
-\chi_{q, 2}^{-} \\
1
\end{array}\right)+b_{11} e_{q, 3}^{-}\left(\begin{array}{c}
\chi_{q, 3}^{-} \\
1 \\
0 \\
0
\end{array}\right)+b_{12} e_{q, 3}^{-}\left(\begin{array}{c}
0 \\
0 \\
-\chi_{q, 3}^{-} \\
1
\end{array}\right),
\end{gathered}
$$

where we used the same shorthand notation as defined in Eq. (10) with $k \rightarrow q$ and $\theta \rightarrow \phi$.

In region $3\left(x>\frac{L}{2}\right)$, only propagation away from the barrier is allowed and all terms need to be finite as $x \rightarrow \infty$. We find that three of the $k_{x}$ from Eq. (8) satisfy these conditions $k_{x, 1}^{+}, k_{x, 2}^{-}$and $k_{3}^{+}$. The ansatz for the wavefunction in this region therefore takes the form

$\Phi_{3}(x)=t_{1} e_{k, 1}^{+}\left(\begin{array}{c}\chi_{k, 1}^{+} \\ 1 \\ 0 \\ 0\end{array}\right)+t_{2} e_{k, 1}^{+}\left(\begin{array}{c}0 \\ 0 \\ -\chi_{k, 1}^{+} \\ 1\end{array}\right)+t_{3} e_{k, 3}^{+}\left(\begin{array}{c}-\chi_{k, 3}^{+} \\ 1 \\ 0 \\ 0\end{array}\right)+t_{4} e_{k, 3}^{+}\left(\begin{array}{c}0 \\ 0 \\ \chi_{k, 3}^{+} \\ 1\end{array}\right)+t_{5} e_{k, 2}^{-}\left(\begin{array}{c}-\chi_{k, 2}^{-} \\ 1 \\ 0 \\ 0\end{array}\right)+t_{6} e_{k, 2}^{-}\left(\begin{array}{c}0 \\ 0 \\ \chi_{k, 2}^{-} \\ 1\end{array}\right)$.

We can now determine the transmission coefficients $t_{i}$, the coefficients $a_{i}$ and $b_{i}$ from the requirement that the wavefunctions and their first and second derivatives in different regions are continuous at the two boundaries 
$x=-\frac{L}{2}$ and $x=\frac{L}{2}$. Continuity of the spinor wavefunctions and their first and second derivatives at each junction interface reads as follows

$$
\begin{aligned}
& \Phi_{1}\left(-\frac{L}{2}\right)=\Phi_{2}\left(-\frac{L}{2}\right) ; \quad \Phi_{2}\left(\frac{L}{2}\right)=\Phi_{3}\left(\frac{L}{2}\right) \\
& \left.\partial_{x} \Phi_{1}(x)\right|_{x=-\frac{L}{2}}=\left.\partial_{x} \Phi_{2}(x)\right|_{x=-\frac{L}{2}} \\
& \left.\left.\partial_{x} \Phi_{2}(x)\right|_{x=\frac{L}{2}}=\partial_{x} \Phi_{3}(x)\right)_{x=\frac{L}{2}} \\
& \left.\partial_{x}^{2} \Phi_{1}(x)\right|_{x=-\frac{L}{2}}=\left.\partial_{x}^{2} \Phi_{2}(x)\right|_{x=-\frac{L}{2}} \\
& \left.\partial_{x}^{2} \Phi_{2}(x)\right|_{x=\frac{L}{2}}=\left.\partial_{x}^{2} \Phi_{3}(x)\right|_{x=\frac{L}{2}}
\end{aligned}
$$

To avoid lengthy and cumbersome expressions, we do not present the resulting system of equations. We will also not include such expressions in later sections.

Next, one has to compute transmission probabilities, which can be done through currents. The probability current density in $x$-direction for a state $\psi$ can be computed as follows

$$
J_{x}=\psi^{\dagger} \frac{\partial H(k)}{\partial k_{x}} \psi
$$

where

$$
\frac{\partial H(k)}{\partial k_{x}}=3 \tau_{z} \otimes\left[\left(k_{x}^{2}-k_{y}^{2}\right) \sigma_{x}-2 k_{x} k_{y} \sigma_{y}\right] .
$$

We may therefore use Eq. (14) to compute explicitly the incident current from the wave coming from $x=-\infty$ as

$$
J_{x}^{\mathrm{inc}}=6 k_{x, 1}^{+}\left[\left(k_{x, 1}^{+}\right)^{2}+(k \sin \theta)^{2}\right]^{\frac{1}{2}}
$$

and for the transmitted wave in the asymptotic limit $x \rightarrow+\infty$ as

$$
J_{x}^{\mathrm{tra}_{1,2}}=6 k_{x, 1}^{+}\left[\left(k_{x, 1}^{+}\right)^{2}+(k \sin \theta)^{2}\right]^{\frac{1}{2}}\left|t_{1,2}\right|
$$

The transmission coefficient in this limit is then defined as

$$
T=\frac{\left|J_{x}^{\text {tra }}\right|}{\left|J_{x}^{\text {inc }}\right|}
$$

which allows us to compute transmission coefficient

$$
T_{1,2}=\left|t_{1,2}\right|^{2}
$$

These steps allow us to compute transmission coefficients numerically.

\subsubsection{Numerical Results}

Next we present our results for this simplified version of the Hamiltonian. As discussed previously we choose our incoming wave to have a wavevector $\mathbf{k}=k(\cos \theta, \sin \theta)$. This means that we can either set $a_{1}=1$ or $a_{2}=1$ to have a wave with these properties. Because the two blocks in the Hamiltonian differ only in sign and do not mix we find that the transmission coefficients for these cases fulfill the condition $T_{1}=T_{2}$. Therefore we only present plots for $T_{1}$.

The transmission as a function of the wave vector $k$ is shown in Fig. 3. 


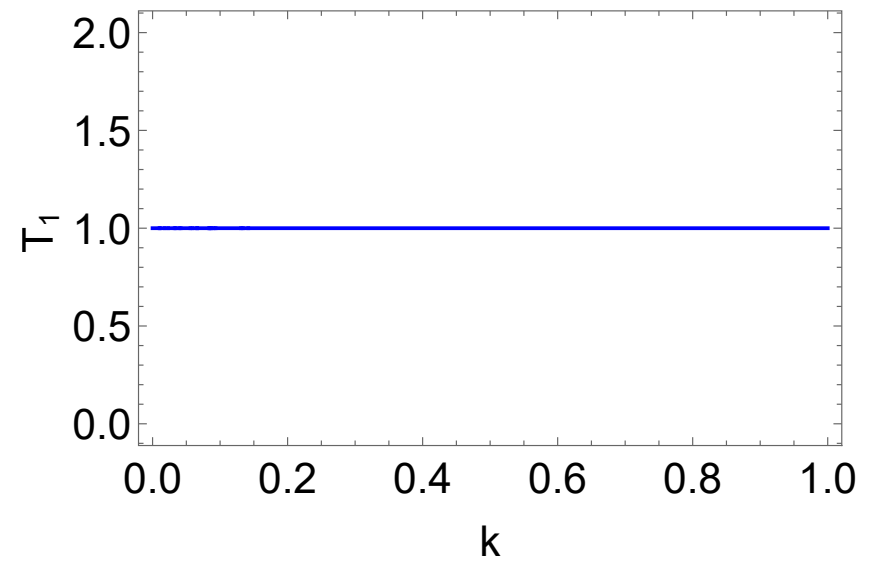

(a) For a potential barrier of width $L=2$ and $u_{0}=2$ at normal incidence $\theta=0$.

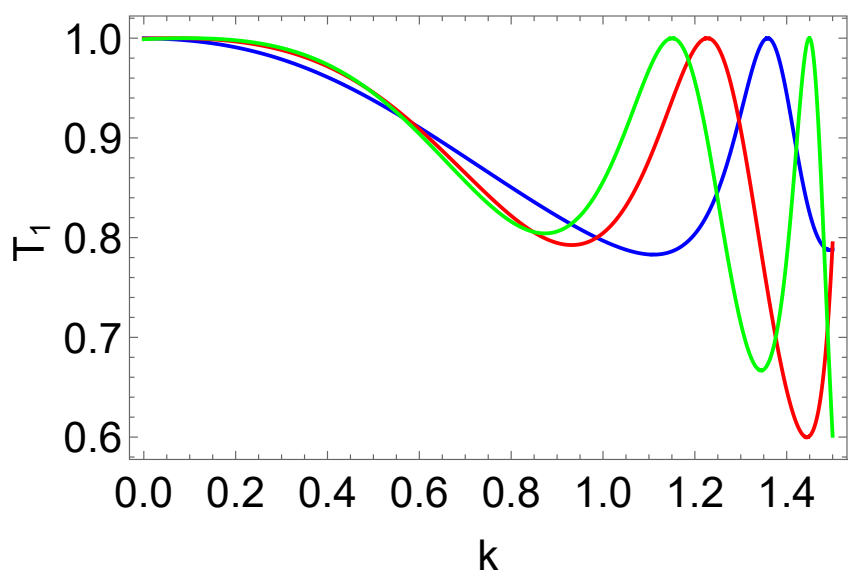

(b) $u_{0}=4$ and small incident angle $\theta=0.1 \mathrm{rad}$. The results were plotted for various values of the barrier width $L=2$ (blue line), $L=5$ (red line), $L=6$ (green line).

Figure 3: (color online) The transmission amplitude $T_{1}$ as a function of the incident wavevector amplitude $k$.

We find that for normal incidence as seen in Fig. 3a much like in the single layer graphene case, there is unit transmission up to numerical accuracy, which is commonly referred to as Klein tunneling. It is interesting to note that one block of the Hamiltonian has the same form as the Hamiltonian for ABC stacked trilayer graphene that is described in [59]. Therefore this result also applies to trilayer graphene in that approximation. One should also note that this contrasts with the result in $\mathrm{AB}$ bilayer graphene, where for normal incidence there is no Klein tunneling. This motivates the question of whether Klein tunneling in multilayer graphene of this stacking type is an effect that appears for an odd number of layers and disappears for an even number of layers, that is, whether it is an even-odd effect with respect to the number of layers.

For a small incident angle keeping all other parameters fixed the result is shown in Fig. $3 \mathrm{~b}$ and we find that the transmission amplitude $T_{1}$ as a function of the incident wavevector amplitude $k$ first decreases and then begins to oscillate. As we increase the barrier width $L$ we observe that the oscillations set in earlier.

We now turn to the transmission amplitude $T_{1}$ as a function of the width of the potential barrier $L$ in Fig. 4a.

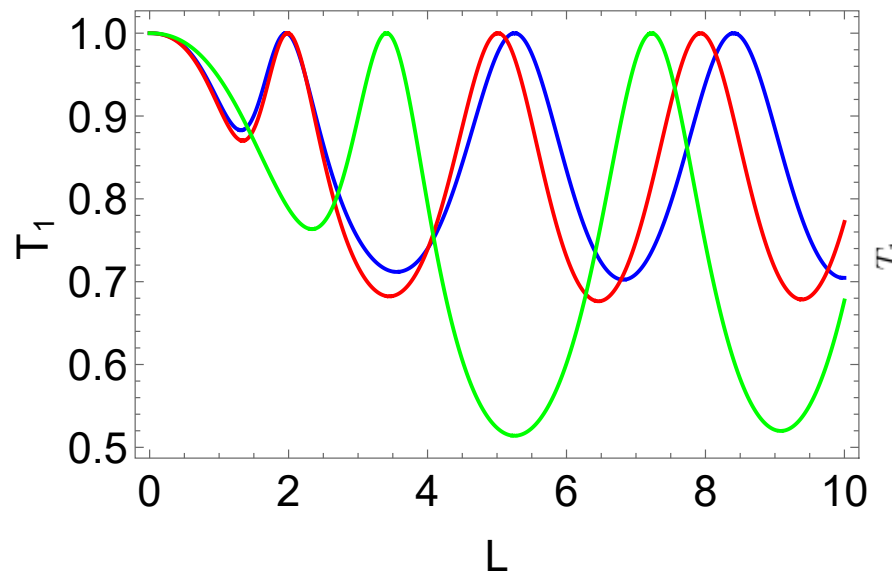

(a) Plot as a function of $L$

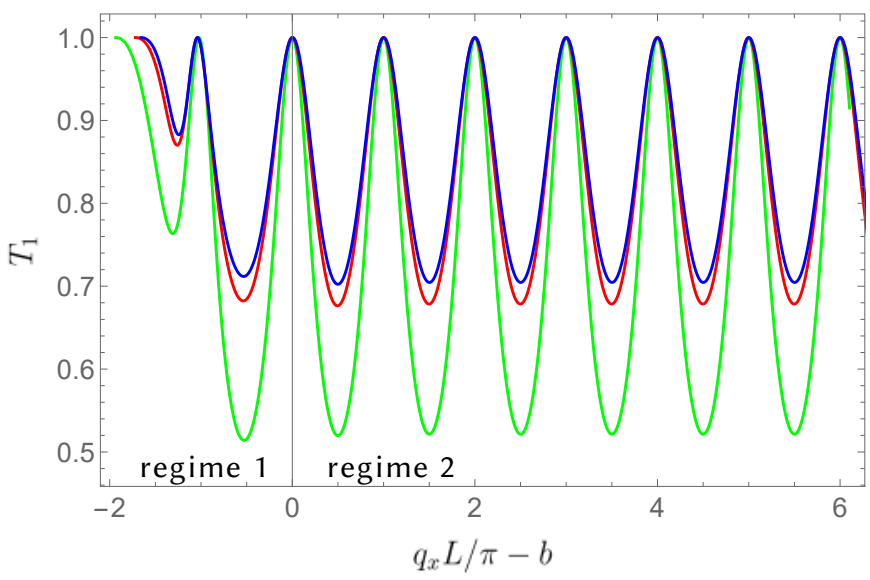

(b) Plot as a function of $q_{x} L / \pi-b$, where $b$ is a bias.

Figure 4: (color online) The transmission coefficient $T_{1}$ at incident angle $\theta=0.1$. We have plotted separate curves for $k=1, u_{0}=2$ (blue line), $k=1.2, u_{0}=3$ (red line) and $k=1.5, u_{0}=4$ (green line).

Like in the previous plot 3a we find resonances and it is not immediately obvious from Fig. 4a why they arise. The physical mechanism behind oscillations can, however, easily be understood if we choose the axis of our plot slightly differently as $q_{x} L / \pi-b$ instead of $L$, where $q_{x}$ is the momentum of the propagating wave inside the barrier and $b$ is a bias that was introduced for each curve to obtain a more lucid result. First we may realize that there are two regimes. In regime 1 we do not observe much structure. This is because this regime corresponds to a not very 
thick barrier where both the exponentially decaying and oscillatory modes $q_{x, i}$ penetrate the barrier. This makes them equally important and it is difficult to disentangle their effects. It is important to note that the length of this regime also depends on the details of the barrier and incoming wave. This is why it was necessary to introduce a bias $b$ for each of the curves to render the plot for regime 2 more lucid. In regime 2 we find that the resonances fulfill $\left(q_{x}^{i}-q_{x}^{i+1}\right) L=\pi$, where $q_{x}^{i}$ labels the $i$-th wave maximum. This requirement for constructive interference inside the barrier is commonly referred to as a Fabry-Pérot type resonance condition.

Finally in Fig. 5 we obtain a polar plot of the transmission as a function of the incident angle $\theta$.

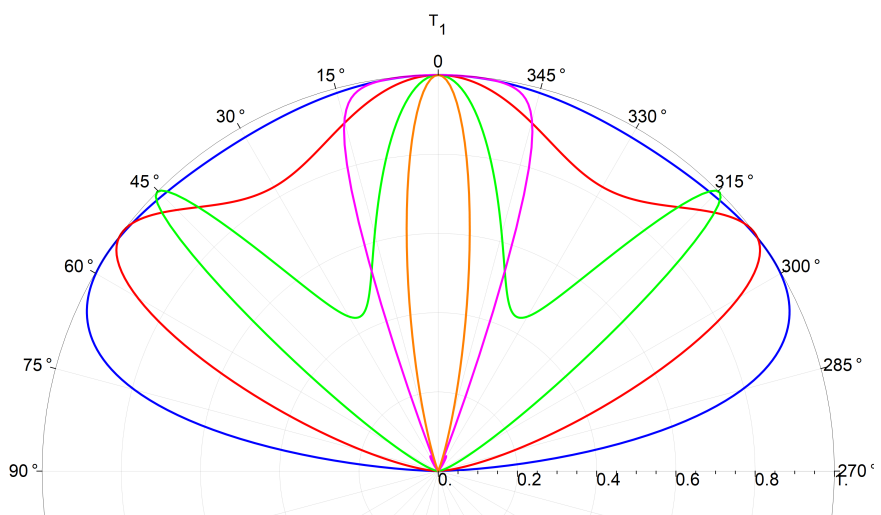

Figure 5: (color online) The transmission coefficient $T_{1}$ as a function of the incident angle $\theta$ for $u_{0}=2$ and $k=1$ and for five different potential barrier widths $L=0.2$ (blue line), 0.5 (red line), 1 (green line), 2 (magenta line), 3 (orange line).

We find that the results are similar to those obtained for single layer graphene but are fundamentally different from those of $\mathrm{AB}$ bilayer graphene that shows anti-Klein tunneling at normal incidence. We have also numerically computed $\left.\frac{d^{2} T_{1}}{d \theta^{2}}\right|_{\theta=0}$ and thereby found that $T_{1}-1 \propto \theta^{2}+\mathcal{O}\left(\theta^{4}\right)$. The Klein tunneling for this case is therefore just as pronounced as in the case of graphene and not fundamentally stronger like in one of the cases we will consider later.

\subsection{Isotropic $\left(k_{z} \neq 0\right)$ limit}

In this section we will discuss the case of a cubic Dirac semi-metal that is still isotropic in the plane but has a large enough extension in $z$-direction to allow for quantized non-zero momenta $k_{z}$. We may define another unitless quantity $\delta=k_{z} / 2$, that can be interpreted as a mass-like term in the original Hamiltonian, as we will see shortly. This is similar to how in Kaluza-Klein theory with an additional periodic space direction the boundary conditions lead to a quantized momentum associated with that direction that is interpreted as a mass term. Similarly, this quantized momentum can then be interpreted as a mass term as we will see. In this case the Hamiltonian takes the form

$$
H(k)=\left(\begin{array}{cccc}
u+\delta & \hat{k}_{+}^{3} & 0 & 0 \\
\hat{k}_{-}^{3} & u-\delta & 0 & 0 \\
0 & 0 & u-\delta & -\hat{k}_{+}^{3} \\
0 & 0 & -\hat{k}_{-}^{3} & u+\delta
\end{array}\right) .
$$

The eigenvalue equation $H(k) \Psi=\epsilon \Psi$ can be solved in the different regions of space and leads to the eigenvalues

$$
\epsilon_{j}=s_{j} \sqrt{k^{6}+\delta^{2}}+u_{j}
$$

where each eigenvalue is twice degenerate, the sign function $s_{j}=\operatorname{sgn}\left(\epsilon_{j}-u_{j}\right)$ and $j=1,2,3$ is labeling the three spatial regions.

The band structure for the different spatial regions is shown in Fig. 6. 

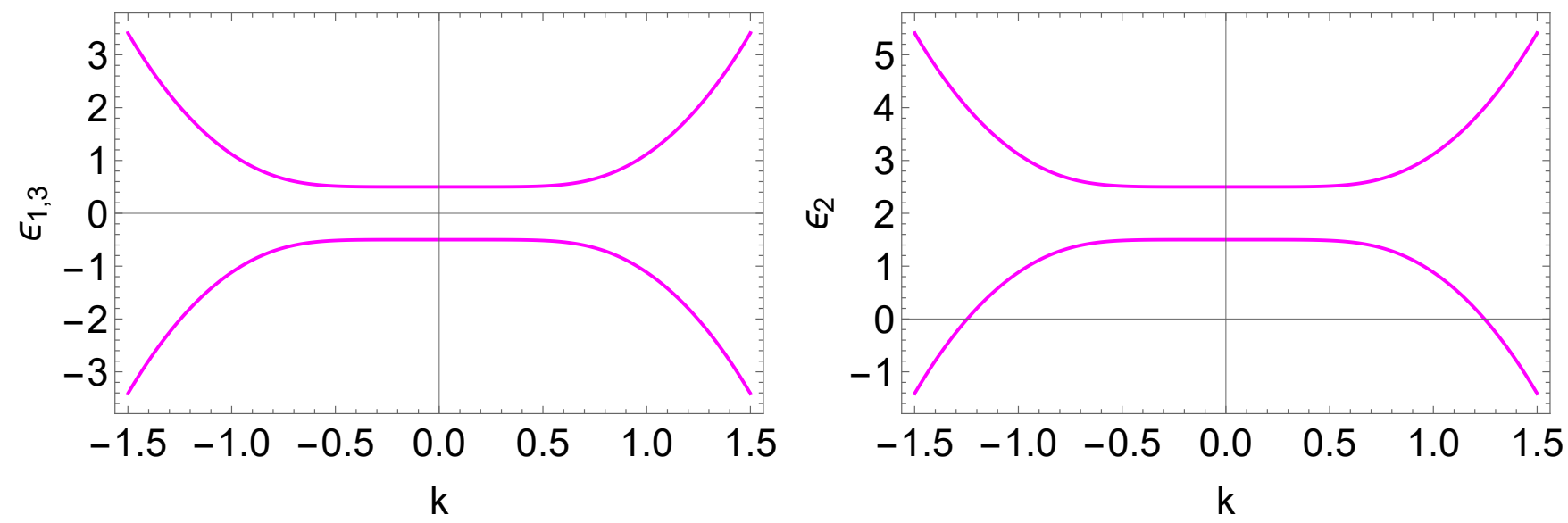

Figure 6: (color online) The dispersion relation in the three different spatial regions as a function of the incident wavevector amplitude $k$ for the value of energy gap $\delta=0.5$. The left panel shows regions 1 and 3 and the right panel shows region 2 with $u_{0}=2$.

We find that, much like a mass term, $\delta$ leads to a gap in the energy spectrum. However, it differs from the dispersion of relativistic particles or electrons in a single layer transition metal dichalcogenide [50] in that the dispersion near $k=0$ is more flat - we quantify this to be $\epsilon \propto k^{6}$ near $k=0$ unlike the relativistic case where $\epsilon \propto k^{2}$.

Much like in the previous section it is advantageous to recast the problem as a cubic generalized eigenvalue problem for $k_{x}$

$$
\left[k_{x}^{3} \tau_{z} \otimes \sigma_{x}+3 i k_{y} k_{x}^{2} \tau_{z} \otimes \sigma_{y}-3 k_{y} k_{x} \tau_{z} \otimes \sigma_{1}-i k_{y}^{3} \tau_{z} \otimes \sigma_{y}+\left(u_{j}-\epsilon\right) \mathbb{1}_{2} \otimes \mathbb{1}_{2}+\delta \tau_{z} \otimes \sigma_{z}\right] \psi=0,
$$

where $j=1,2,3$ labels the different spatial regions. With the wavevector amplitude $k=\left(\epsilon^{2}-\delta^{2}\right)^{\frac{1}{6}}$ we find that the solutions for $k_{x}$ are still given by Eq. (8).

For brevity we don't repeat the analysis of wavefunctions for the different regions because it is almost the same as in the massless case. The results are given in appendix Appendix B.1.

Like previously we may now use Eq. (13) and set $a_{1}=a_{2}=1$ to solve the remainder of the problem numerically.

\subsubsection{Numerical Results}

Next we present our numerical results for the transmission amplitudes $T_{1,2}$. Like what we did previously, we will restrict our discussion to $T_{1}$ because $T_{1}=T_{2}$ by symmetry and set $a_{1}=1$ to determine the properties of the incoming wave. Since we have added a new mass term $\delta$ to the Hamiltonian, we will first and foremost be concerned with its influence. The result for a barrier width $L=2$ and normal incidence are presented in Fig. 7 .

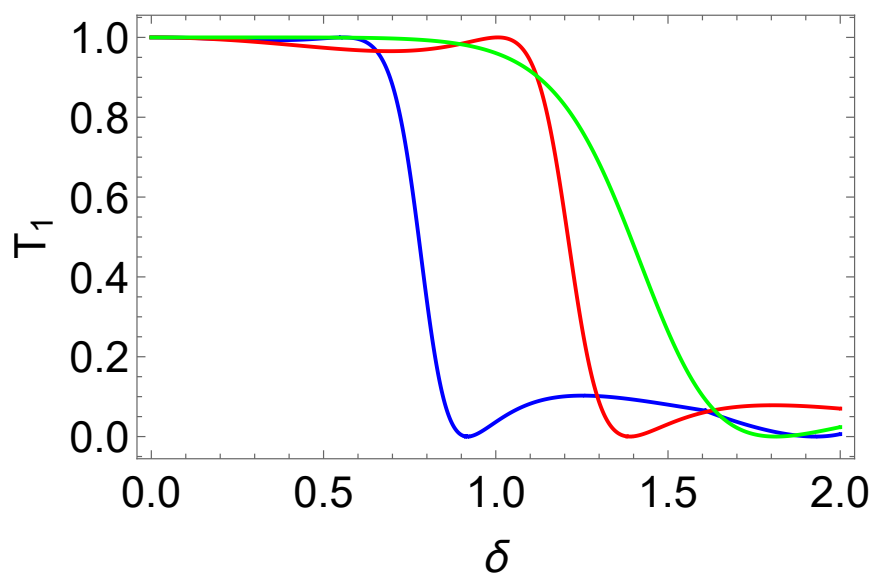

Figure 7: (color online) The transmission coefficient $T_{1}$ for normal incidence $\theta=0$ as a function energy gap $\delta$ for a barrier width $L=2$. We chose other values $u_{0}=3.5, k=1$ (blue line), $u_{0}=5, k=1.2$ (red line) and $u_{0}=7, k=1.5$ (green line).

We find that the mass-like term $\delta$ suppresses tunneling like one would expect from the semi-classical intuition that mass suppresses quantum phenomena like tunneling. We also find that there are certain values of $\delta$ where the 
transmission probability despite increasing the mass-like term rises again and for some specific values even reaches unity again. This is likely due to Fabry-Pérot type interference effects inside the barrier, which we will confirm shortly.

To gain further intuition let us now study $T_{1}$ as a function of the incident wavevector amplitude $k$ as shown in Fig. 8 .

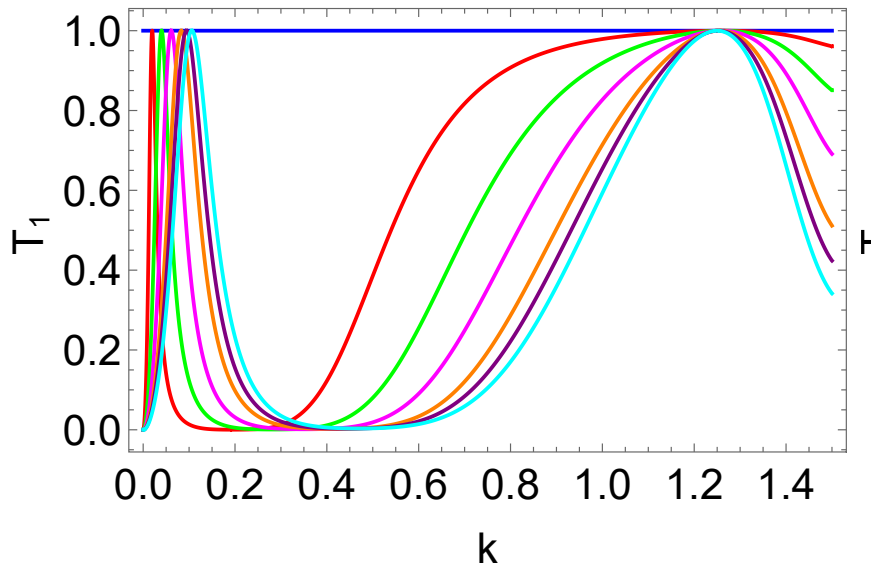

(a) Incidence at $\theta=0 \mathrm{rad}$.

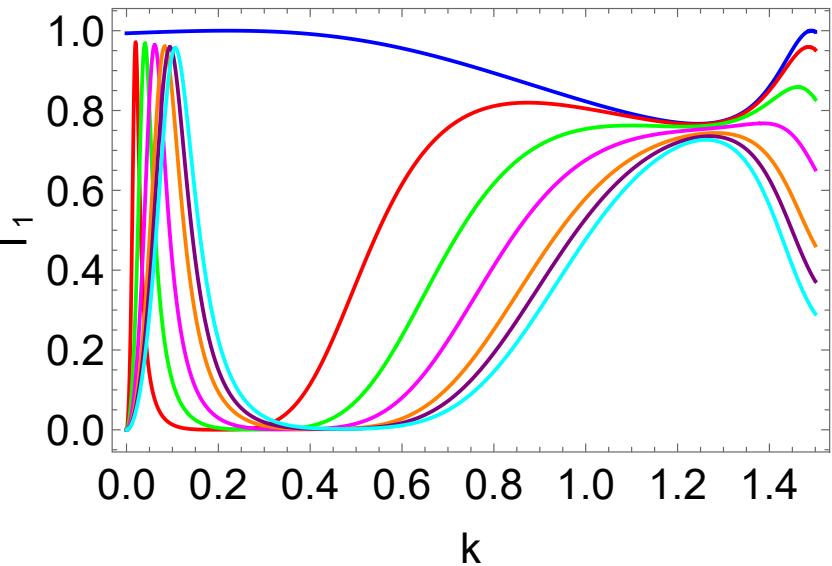

(b) Incidence at $\theta=0.1 \mathrm{rad}$.

Figure 8: (color online) The transmission amplitude $T_{1}$ as a function of the incident wavevector amplitude $k$ for $u_{0}=4$ and barrier width $L=2$. We have plotted separate curves for different energy gaps $\delta=0$ (blue line), $\delta=0.1$ (red line), $\delta=0.2$ (green line), $\delta=0.3$ (magenta line), $\delta=0.4$ (orange line), $\delta=0.45$ (purple line) and $\delta=0.5$ (cyan line).

We find that the inclusion of the mass terms leads to a gap in the transmission amplitudes in the region of $0.2 \lesssim k \lesssim 0.8$. This transmission gap is again best explained semi-classically. A particle with increasing mass will behave increasingly classical. Classical particles cannot tunnel through a barrier. Therefore with increasing mass one observes a transmission gap. We also see that for incidence that is not normal the transmission amplitude does not reach unity again in the massive case but the resonances appear at approximately the same spots as the massless case. They are therefore related to the same physical phenomenon.

To understand this phenomenon better and relate it to the Fabry-Pérot interferrometer we again plot the transmission amplitude $T_{1}$ as a function of the width of the potential barrier $L$ in Fig. 9 .

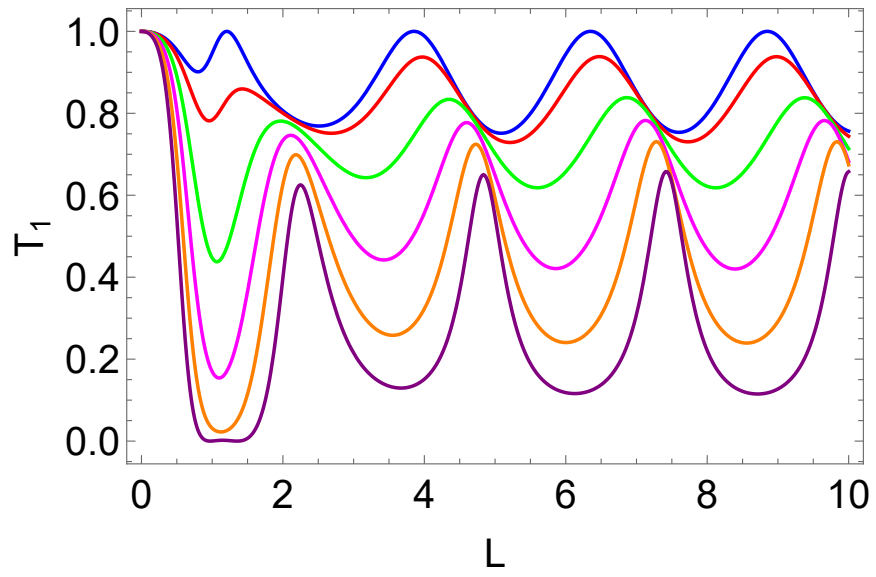

(a) Plot as function of $L$

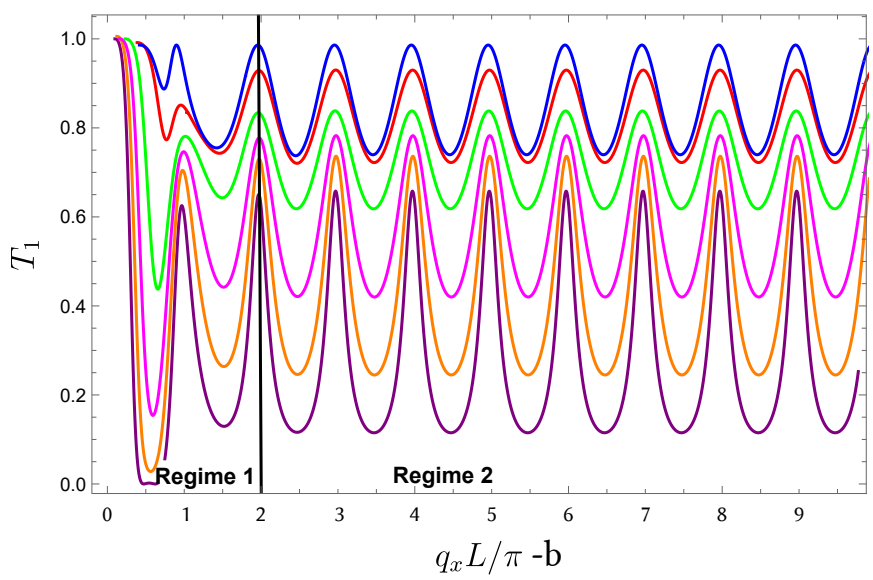

(b) Plot as a function of $q_{x} L / \pi-b$

Figure 9: (color online) The transmission amplitude $T_{1}$ as a function of barrier width for incident wavevector amplitude $k=1, u_{0}=3$ and $\theta=0.1 \mathrm{rad}$. We have plotted separate curves for energy gaps $\delta=0$ (blue line), 0.1 (red line), 0.2 (green line), 0.3 (magenta line), 0.4 (orange line) and $\delta=0.5$ (purple line).

We first observe that oscillations as a function of barrier thickness $L$ become increasingly sharp peaks as we increase the magnitude of the mass term, $\delta$. We observe a suppression of tunneling except at certain specific 
resonant values.

To understand the physical mechanism underlying these resonances in Fig. 9b we plot the transmission coefficient as a function of $q_{x} L / \pi-b$, where $b$ is a bias that was introduced to obtain a more lucid plot.

Like in the mass-less case we find again that there are two regimes. Regime 1 does not have much structure. This is because this regime corresponds to a thin barrier where both the exponentially decaying and oscillatory modes $q_{x, i}$ contribute equally inside the barrier. In regime 2 we find that the resonances fulfill $\left(q_{x}^{i}-q_{x}^{i+1}\right) L=\pi$, where $q_{x}^{i}$ labels the $i$-th wave maximum. This is similar to previous section and this requirement is called the Fabry-Pérot resonance condition.

In Fig. 10 we show polar plots of the transmission coefficient $T_{1}$ as a function of the incident angle $\theta$.

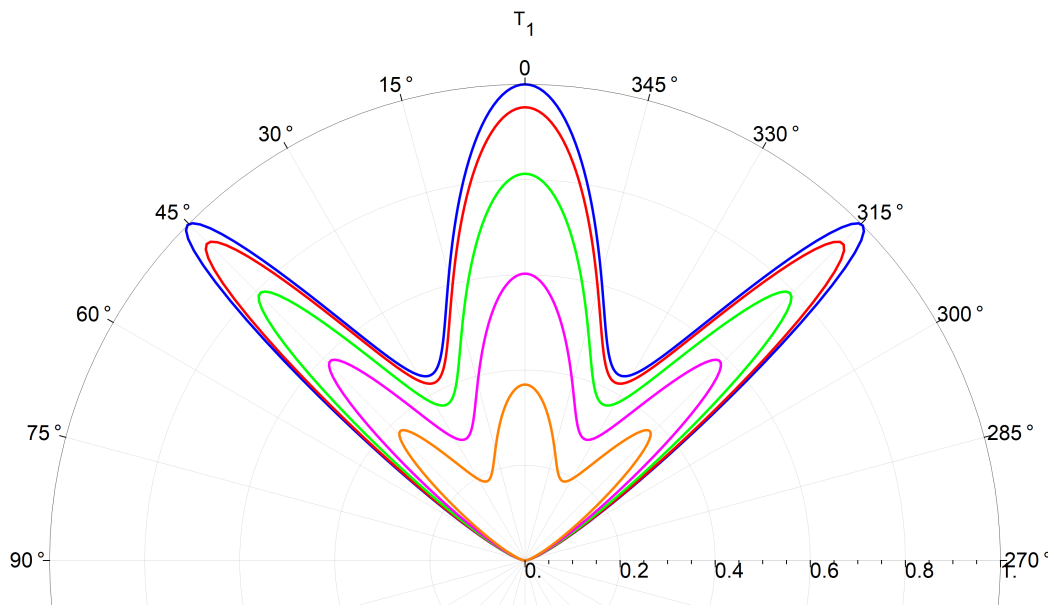

Figure 10: (color online) The angular dependence of the transmission coefficient $T_{1}$ for $u_{0}=2, k=1$ and barrier width $L=1$ for five values of the energy gap $\delta=0$ (blue line), 0.1 (red line), 0.2 (green line), 0.3 (magenta line), 0.4 (orange line).

For the chosen parameters we find that the main effect of the mass-like term, $\delta$, is to weaken the strength of the barrier transmission. This is in full agreement with the semi-classical intuition one has that a heavier particle is "less quantum" and therefore should be less susceptible to exhibit quantum effects such as tunneling.

\subsection{Anisotropic 2D $\left(k_{z}=0\right)$ limit}

In this section we will consider the case that is anisotropic in the $x$-y plane, where $v_{y}=\lambda v_{x}=\lambda v$. We consider again the case of thin material with $k_{z}=0$. Much like in the previous cases it is advantageous to define unitless quantities via Eq. (5). The Hamiltonian of the system is given by

$$
H(k)=\left(\begin{array}{cccc}
0 & \hat{k}_{1} & 0 & 0 \\
\hat{k}_{1}^{\dagger} & 0 & 0 & 0 \\
0 & 0 & 0 & -\hat{k}_{1} \\
0 & 0 & -\hat{k}_{1}^{\dagger} & 0
\end{array}\right)+u_{j} \mathbb{1}_{4},
$$

where

$$
\hat{k}_{1}=\frac{\lambda+1}{2} \hat{k}_{+}^{3}+\frac{\lambda-1}{2} \hat{k}_{-}^{3} .
$$

For this anisotropic Hamiltonian we find that the energy eigenvalues

$$
\epsilon_{j}= \pm \sqrt{\lambda^{2} k_{x}^{2}\left(k_{x}^{2}-3 k_{y}^{2}\right)^{2}+\left(k_{y}^{3}-3 k_{x}^{2} k_{y}\right)^{2}}+u_{j},
$$

also have an anisotropic energy dispersion relation that has a sixfold rotational symmetry as shown in Fig. 11. 


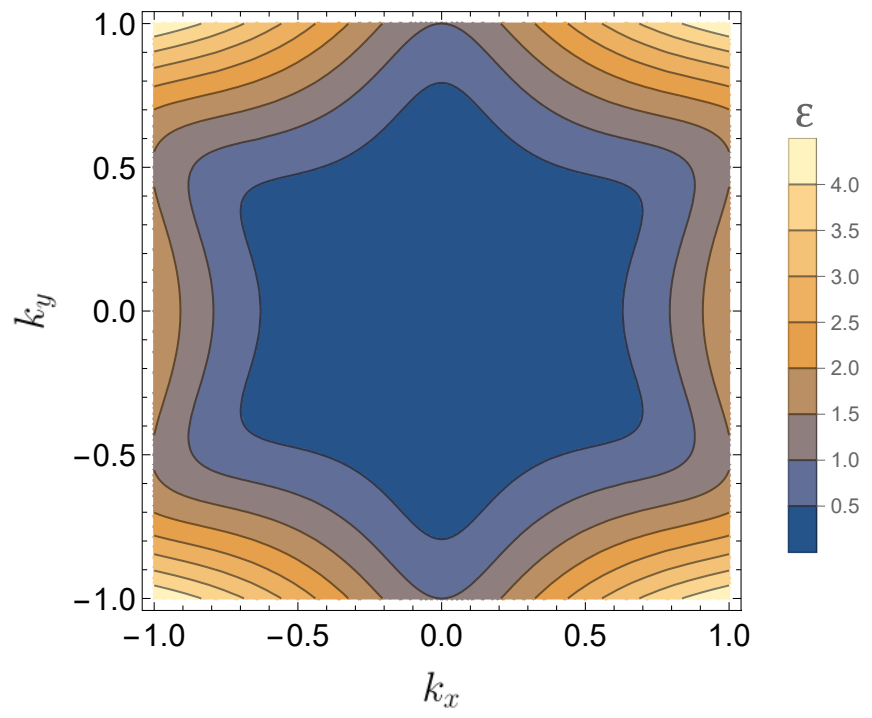

(a) Contour plot of the positive energy band.

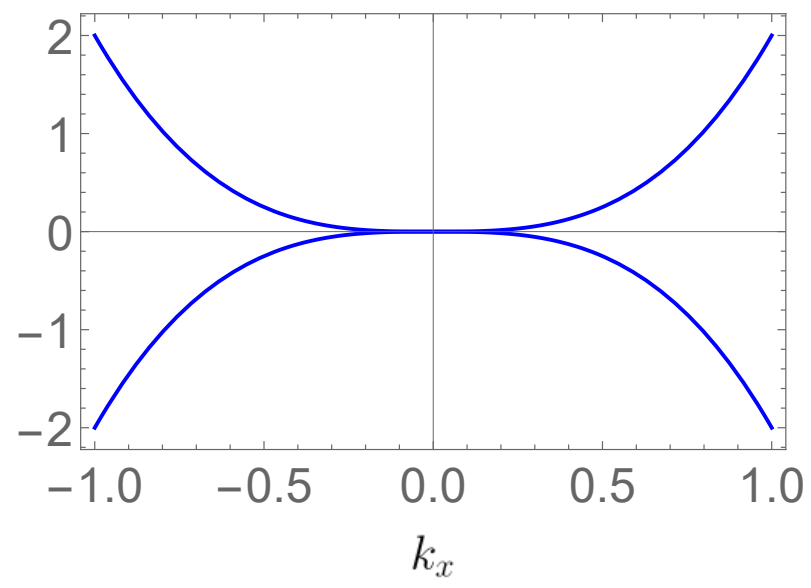

(b) Slice at $k_{y}=0$ for both energy solutions

Figure 11: (color online) Plots of the dispersion relation in region 1 for $\lambda=2$.

We find that even though rotational symmetry is broken, the system is still gap-less.

\subsubsection{Solution of the tunneling problem}

Before we investigate the tunneling problem in detail let us make an interesting observation about $k_{1}=\lambda k_{x}^{3}+$ $3 i k_{x}^{2} k_{y}-3 \lambda k_{x} k_{y}^{2}-i k_{y}^{3}$. Namely we find that if we make the following transformation

$$
\lambda \rightarrow \frac{1}{\lambda} ; \quad k_{x, y} \rightarrow \lambda^{\frac{1}{3}} k_{x, y}
$$

then up to a relabeling of Pauli matrices the roles of $k_{x}$ and $k_{y}$ in the full Hamiltonian get interchanged, that is, $k_{1} \rightarrow k_{x}^{3}+3 i \lambda k_{x}^{2} k_{y}-3 k_{x} k_{y}^{2}-i \lambda k_{y}^{3}$. This means that the replacements (26) amounts to a hidden symmetry in parameters that allows us to use results for tunneling through a barrier in $x$-direction and without additional effort deduce results for tunneling through a barrier in $y$-direction. One just has to make proper use of the replacements (26). We will therefore, in our analytical treatment restrict ourselves to a potential barrier in $x$-direction but will still be able to compute results for tunneling through a barrier in $y$-direction.

For an incoming plane wave with momentum $\mathbf{k}=(k \cos \theta, k \sin \theta)$ we find that the possible eigenenergies are given by

$$
\epsilon= \pm \frac{1}{\sqrt{2}} \sqrt{k^{6}\left[\left(\lambda^{2}-1\right) \cos (6 \theta)+\lambda^{2}+1\right]},
$$

and each eigenvalue is twice degenerate. We may now determine the allowed momenta $k_{x}$ at this energy in the different regions if we solve the non-linear generalized eigenvalue problem

$$
\left[\lambda k_{x}^{3} \tau_{z} \otimes \sigma_{x}-3 k_{x}^{2} k_{y} \tau_{z} \otimes \sigma_{y}-3 \lambda k_{x} k_{y}^{2} \tau_{z} \otimes \sigma_{x}+k_{y}^{3} \tau_{z} \otimes \sigma_{y}+\left(u_{j}-\epsilon\right) \mathbb{1}_{4}\right] \psi=0,
$$

for $k_{x}$ at fixed energy $\epsilon_{j}$ given in equation (27) and $k_{y}=k \sin \theta$. We find that there are six possible wavevectors $k_{x}$ in region 1, which are given by

$$
\begin{aligned}
& k_{x, 1}^{+}=-k_{x, 1}^{-}=k \cos (\theta) \\
& k_{x, 2}^{+}=-k_{x, 2}^{-}=-\frac{k}{2 \lambda} \operatorname{sign}\left(\gamma_{-}(\theta)\right)\left|f_{+}(\theta)\right|^{1 / 2} e^{i \gamma_{-}(\theta)} \\
& k_{x, 3}^{+}=-k_{x, 3}^{-}=\operatorname{sign}\left(\gamma_{+}(\theta)\right) \frac{k}{2 \lambda}\left|f_{-}(\theta)\right|^{1 / 2} e^{i \gamma_{+}(\theta)},
\end{aligned}
$$

where the sign convention is chosen to allow an easier classification of complex wavevectors.

$$
f_{ \pm}(\theta)=\lambda^{2}(5-7 \cos (2 \theta)) \pm \sqrt{\frac{3}{2}}\left[81-4\left(\lambda^{4}-28 \lambda^{2}+27\right) \cos (2 \theta)+\left(27-5 \lambda^{4}-22 \lambda^{2}\right) \cos (4 \theta)+\lambda^{4}-90 \lambda^{2}\right]^{\frac{1}{2}}-18 \sin ^{2}(\theta)
$$


and $\gamma_{ \pm}(\theta)=\frac{1}{2} \arg \left[f_{ \pm}(\theta)\right]$, with $\sin \left[\gamma_{ \pm}(\theta)\right]>0$. The solutions for $k_{x, i}^{ \pm}$can be real or complex valued depending on the choice of parameters. In Fig. 12 we present a plot of many of the solutions $k_{x, i}^{+}$that are real valued.

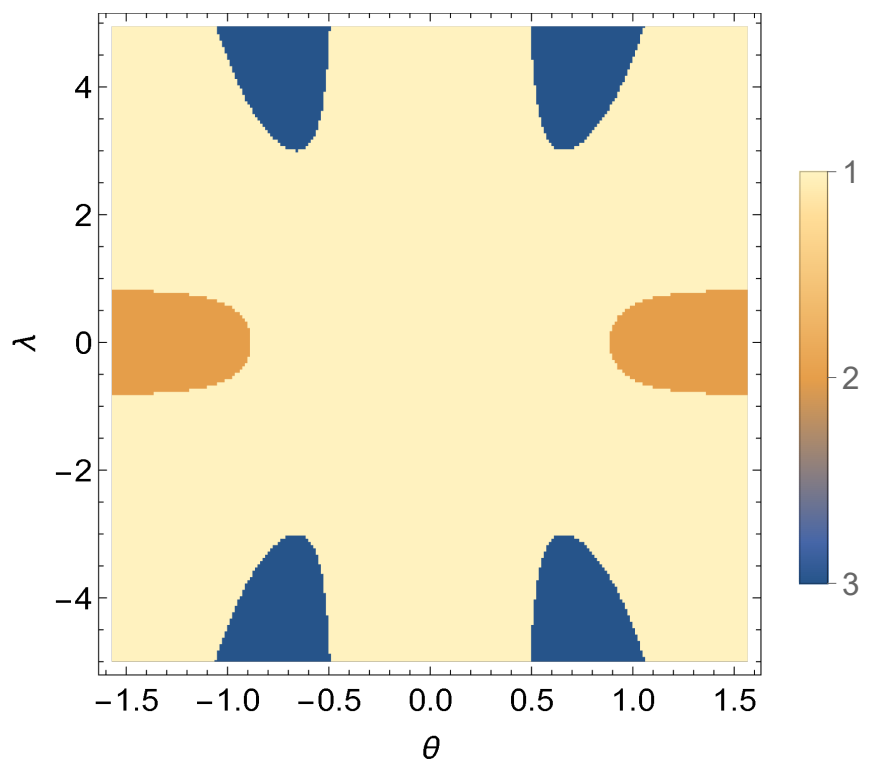

Figure 12: (color online) The number of real valued solutions $k_{x, i}^{+}$as function of anistropy pamarameter $\lambda$ and incident angle $\theta$ (in units of $\mathrm{rad}$ ). This can be interpreted as number of propagating modes that can be detected by a distant detector. It is a tunneling phase diagram.

We find that there are regions with different numbers of real valued solutions. It is useful to recognize that the number of real solutions corresponds to the number of modes that are purely propagating - not exponentially decaying. They therefore can be detected by a distant detector. In this sense the figure shows a kind of tunneling phase diagram of a distant detector.

Because the wave vectors in each region in Fig. 12 have different properties one has to be careful. Particularly, one will require a different ansatz for the wavefunction that takes this into account. In this paper we will restrict ourselves to the light brown regions with only $k_{x, 1}^{+}$as real valued and $k_{x, 2 / 3}^{+}$complex valued. In all further plots later in this section we will restrict our parameters so that each plot point falls into one of these regions.

The ansatz for the wavefunction in the different regions is found similarly to Sec. 3.1 by a discussion of which wavevectors are physically allowed. For brevity we omitted this discussion from the main text and have relegated it to appendix Appendix B.2. With this ansatz for the wavefunction and by matching conditions (13) we may then solve the problem numerically for the different coefficients. We will now turn to these numerical results.

\subsubsection{Numerical Results}

First, in Fig. 13 we present the transmission coefficient $T_{1}$ as a function of the width of the potential barrier $L$. We do this for two different orientations of the barrier to capture the effect of the anisotropy. 


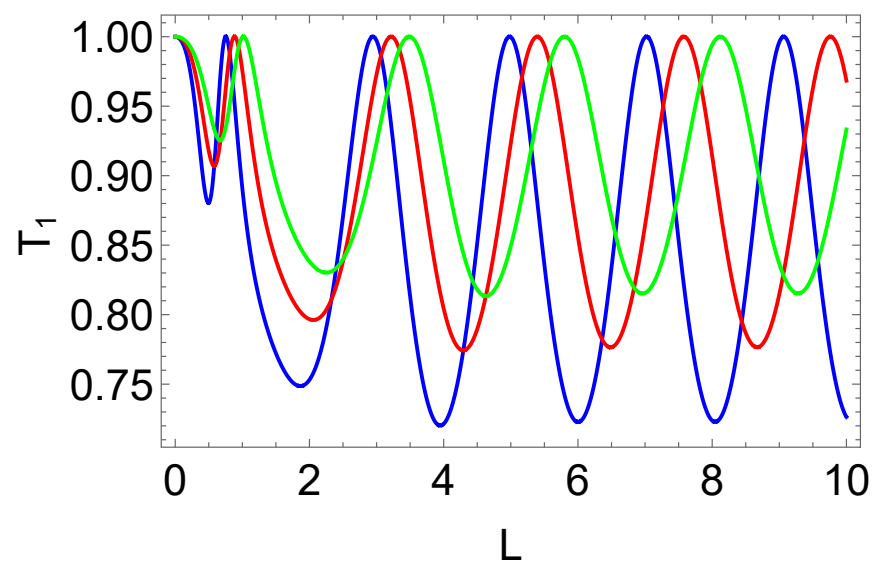

(a) Barrier in $x$-direction

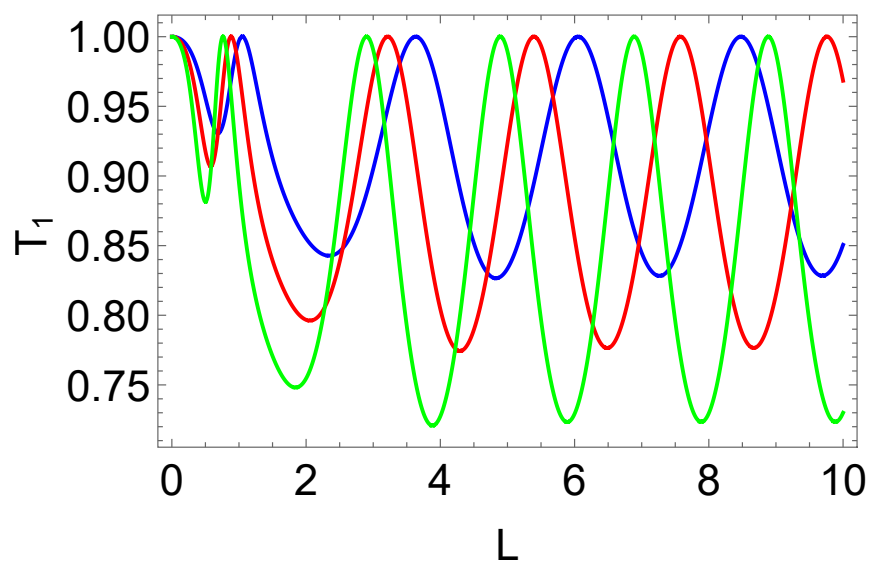

(b) Barrier in $y$-direction.

Figure 13: (color online) The transmission coefficient $T_{1}$ as a function of the barrier thickness $L$ for the incident wavevector amplitude $k=1$, barrier height $u_{0}=4$, incident angle $\theta=0.1 \mathrm{rad}$ and various values of $\lambda=0.85$ (blue line), 1 (red line), 1.15 (green line).

As one would expect we find that the rotational symmetry is broken for $\lambda \neq 1$. We also find that the value of transmission minima changes for different values of $\lambda$. This change in height as we change $\lambda$ can be attributed to the fact that the energy of the incident particle not only depends on $k$ but also on $\lambda$. This means that the curves compare tunneling of particles at different energies. The apparent symmetry between blue and green curves for the two plots is of no deeper physical relevance - it is related to the specific choice of parameters. We also find that the location of the transmission maximum changes for different $\lambda$. This is because $\lambda$ enters into $q_{x, i}$ and therefore also impacts where Fabry-Pérot type resonances occur.

Last, we present the transmission coefficient $T_{1}$ as a function of the incident angle $\theta$ in Fig. 14.

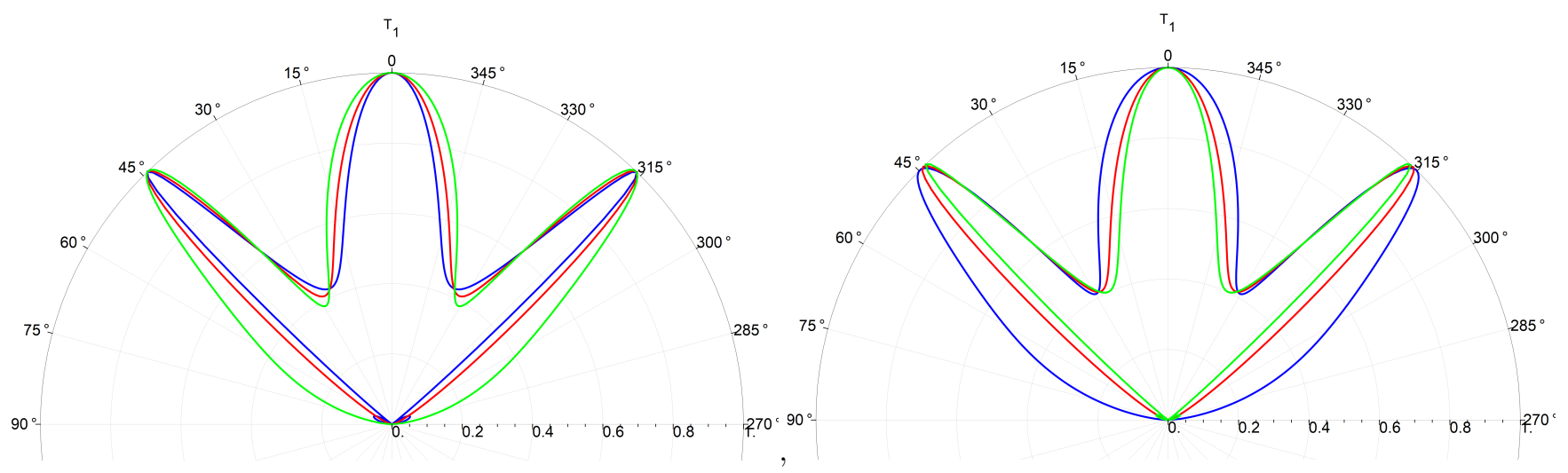

Figure 14: (color online) The transmission coefficient $T_{1}$ as a function of the incident angle $\theta$ for $k=1, u_{0}=10, L=0.2$ and various values of $\lambda=0.85$ (blue line), 1 (red line), 1.15 (green line). The left panel shows results for a barrier located in $x$-direction and the right panel for a barrier in $y$-direction.

Much like in previous plots we find that the most prominent feature is the broken rotational symmetry for $\lambda \neq 1$ - that is the results for a barrier in $x$-direction and a barrier in $y$-direction differ.

\subsection{Anisotropic $\left(k_{z} \neq 0\right)$ limit}

In this section we consider the situation where we have both anisotropy $v_{y}=\lambda v_{x}=\lambda v$ and $k_{z} \neq 0$. The reason for doing this is to see if there is any effect that is uniquely associated with the interplay between anisotropy parameter $\lambda$ and finite momentum $k_{z}$. It is convenient to recast the problem using dimensionless energy $\epsilon=\frac{E}{2 \hbar v}$, 
gap $\delta=\frac{v_{z} k_{z}}{2 v}$ and potential $u_{j}=\frac{V_{j}}{2 \hbar v}$. It may then be described by the Hamiltonian

$$
H(k)=\left(\begin{array}{cccc}
\delta & \hat{k}_{1} & 0 & 0 \\
\hat{k}_{1}^{\dagger} & -\delta & 0 & 0 \\
0 & 0 & -\delta & -\hat{k}_{1} \\
0 & 0 & -\hat{k}_{1}^{\dagger} & \delta
\end{array}\right),
$$

where

$$
\hat{k}_{1}=\lambda \hat{k}_{x}^{3}+3 i \hat{k}_{x}^{2} \hat{k}_{y}-3 \lambda \hat{k}_{x} \hat{k}_{y}^{2}-i \hat{k}_{y}^{3} .
$$

We find that the energies in region 1 are given by

$$
\epsilon_{j}= \pm \sqrt{\lambda^{2} k_{x}^{2}\left(k_{x}^{2}-3 k_{y}^{2}\right)^{2}+\left(k_{y}^{3}-3 k_{x}^{2} k_{y}\right)^{2}+\delta^{2}}
$$

and are twice degenerate.

A plot can be seen in Fig. 15 .

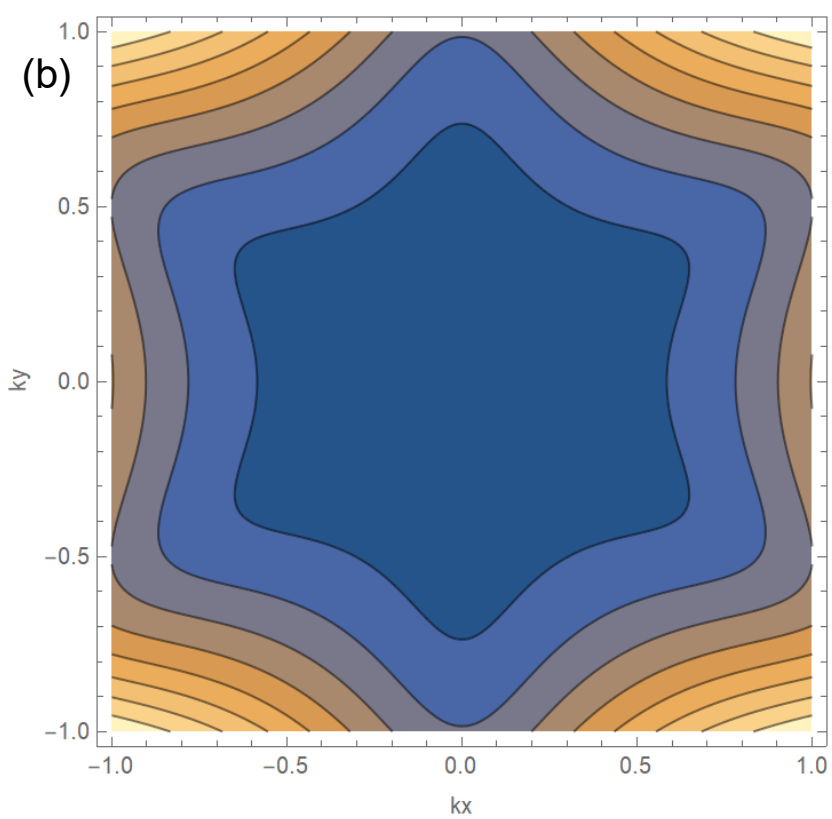

(a) Contour plot of the positive energy band.

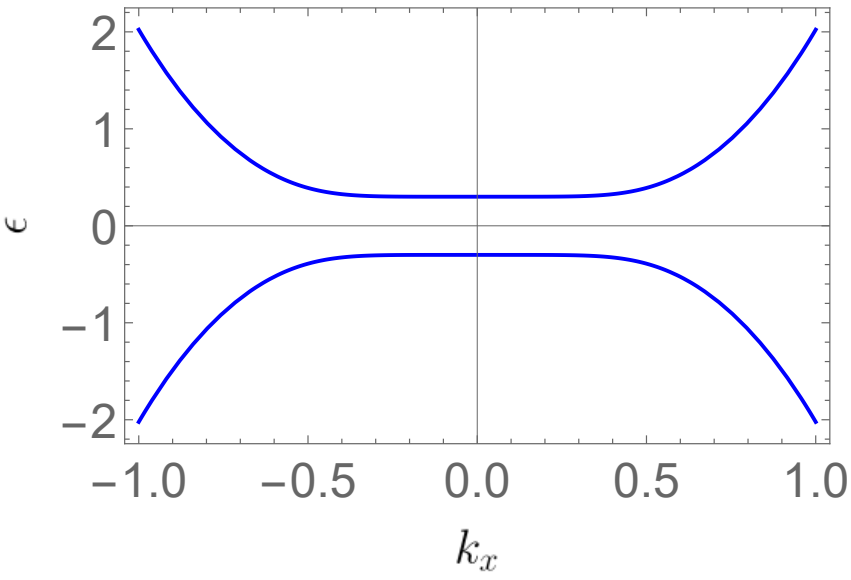

(b) Slice at $k_{y}=0$ of both energy solutions

Figure 15: (color online) Plot of the dispersion in region 1 for $\lambda=2$ and $\delta=0.3$.

We observe a sixfold symmetry and an energy gap.

We will now turn to the solution of the tunneling problem. Here, we may consider in region 1 the case of an incoming wave with wavevector $\mathbf{k}=k(\cos \theta, \sin \theta)$, which is associated with positive energy eigenvalues given as

$$
\epsilon=\frac{1}{\sqrt{2}} \sqrt{2 \delta^{2}+k^{6}\left[\left(\lambda^{2}-1\right) \cos (6 \theta)+\lambda^{2}+1\right]} .
$$

At this energy one may determine the permitted wavevectors that can appear through interaction with the potential barrier. For that, one can interpret the eigenenergy equation as a non-linear generalized eigenvalue problem for $k_{x}$

$$
\left[\lambda k_{x}^{3} \tau_{z} \otimes \sigma_{x}-3 k_{x}^{2} k_{y} \tau_{z} \otimes \sigma_{y}-3 \lambda k_{x} k_{y}^{2} \tau_{z} \otimes \sigma_{x}+k_{y}^{3} \tau_{z} \otimes \sigma_{y}+\delta \tau_{z} \otimes \sigma_{z}+\left(u_{j}-\epsilon\right) \mathbb{1}_{4}\right] \psi=0 .
$$

The possible solutions $k_{x}$ for regions 1 and 3 are again given by Eqs. (29-30) and are therefore independent of $\delta$ but can take both complex and real values. Real valued solutions propagate to infinity, complex valued solutions due to the boundary conditions should only be included if they decay at infinity. Therefore a distant detector will only detect waves corresponding to real-valued solutions of $k_{x}$. Analogously, we may use Fig. 12 to classify the parameter regions that have a certain number of real solutions $k_{x, i}^{+}$. In the following we will again restrict our 
discussion to light brown regions with only one of the $k_{x, i}^{+}$being real valued and will make this assumption for any ansatz wavefunction.

Determining the ansatz wavefunctions in the different spatial regions follows the same approach as in Sec. 3.1 and therefore is again relegated to an appendix Appendix B.3.

We can now solve the problem numerically if we insert the ansatz from appendix Appendix B.3 in (13) and solve for the coefficients.

\subsubsection{Numerical Results}

We will again set $a_{1}=1$ to choose the incoming wave as one with wavevector $\mathbf{k}=(\cos \theta, \sin \theta) k$. This allows us to determine the tunneling amplitude which is then used to compute the transmission amplitude $T_{1}$ shown in Fig. 16 as a function of the width of the potential barrier $L$.

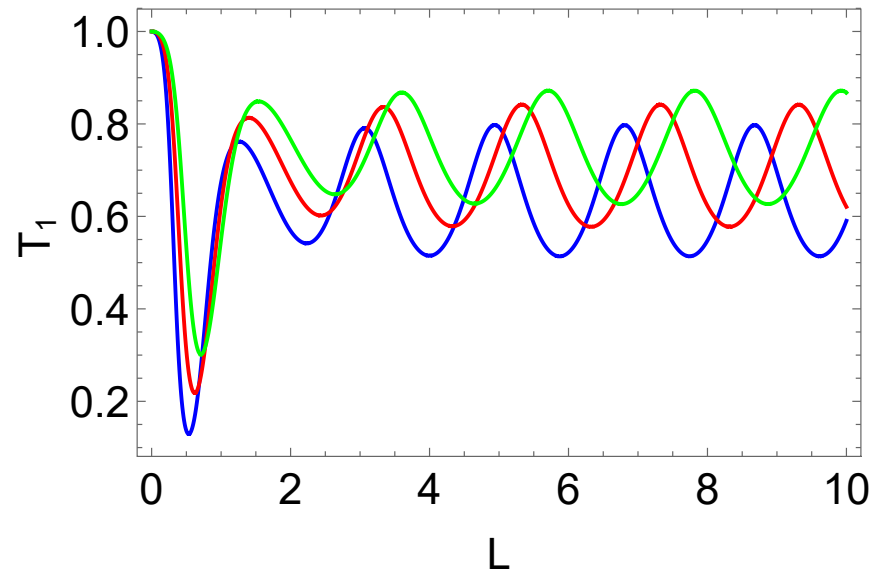

(a) Barrier in $x$-direction

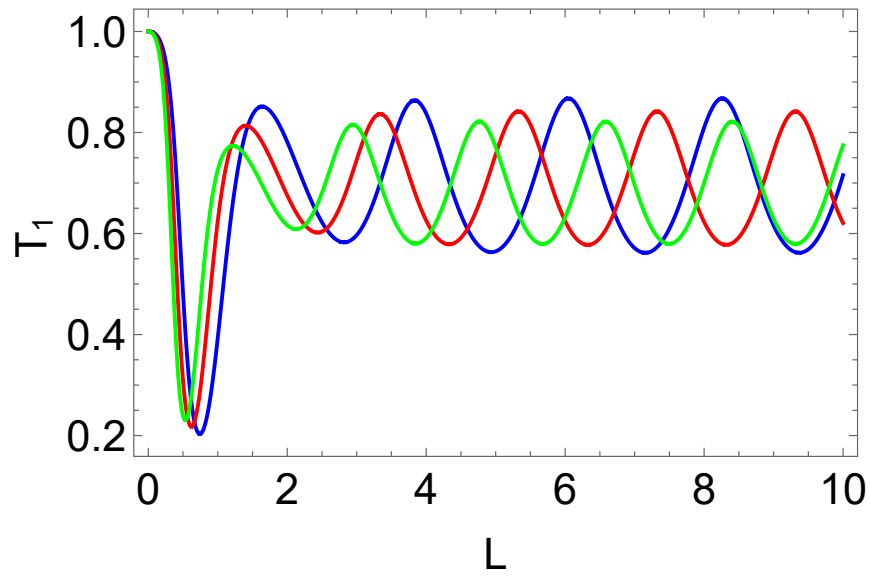

(b) Barrier in $y$-direction

Figure 16: (color online) The transmission coefficient $T_{1}$ as a function of the barrier thickness $L$ for the incident wavevector amplitude $k=1$, barrier height $u_{0}=5$, incident angle $\theta=0.1$ rad and mass term $\delta=0.3$. We plotted various values of $\lambda=0.85$ (blue line), 1 (red line), 1.15 (green line).

We find that transmission probabilities get suppressed by the addition of the mass-like term $\delta$ as one would expect from previous sections and semi-classical intuition which dictates that a mass terms suppresses quantum effects such as tunneling. Addition of the anisotropy $\lambda \neq 1$ breaks rotational symmetry, however, there does not appear to give rise to any special effect that is unique to the coexistence of both anisotropy and mass-like term. The oscillations are again due to a Fabry-Perot-type resonance condition similar to previous sections.

Finally, in Fig. 17 we show the transmission coefficient $T_{1}$ as a function the incident angle $\theta$.

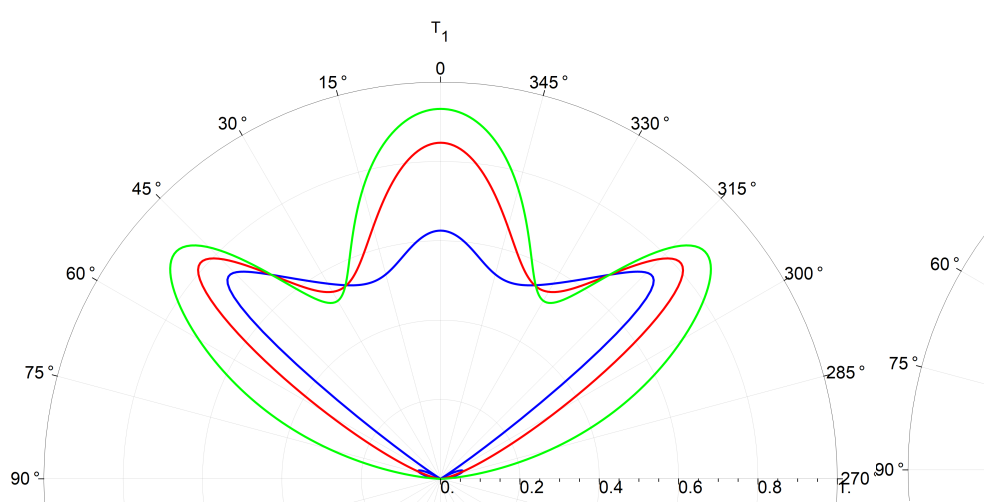

(a) Barrier in $x$-direction

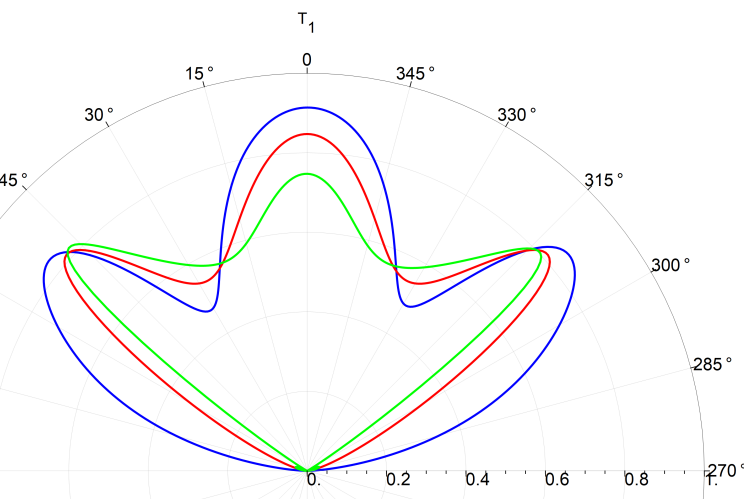

(b) Barrier in $y$-direction

Figure 17: (color online) The transmission coefficient $T_{1}$ as a function of the incident angle $\theta$ for a barrier width $L=0.5$, barrier height $u_{0}=3$, wavevector amplitude $k=1, \delta=0.3$ and various values of $\lambda=0.85$ (blue line), 1 (red line), 1.15 (green line). 
We observe that much like in all other plots the mass-like term $\delta$ leads to smaller maximum values of transmission coefficient $T_{1}$ while the addition of the anisotropy $\lambda \neq 1$ breaks the rotational symmetry. We were unable to find an effect that uniquely depends on the interplay between $\lambda$ and $\delta$. It seems that if there exists such an effect then it might likely occur at values of $\lambda$ and $\theta$ that give rise to more than one real solution. That is they may occur in regions of Fig. 12 that are dark brown or blue.

\subsection{Bulk transmission through a potential along z-direction}

In this section we consider a bulk cubic Dirac semi-metal and put a potential barrier along the $z$-direction rather than in the plane of the cubic dispersion. That is we consider the Hamiltonian

$$
H=\left(\begin{array}{cccc}
\nu \hat{k}_{z} & k_{x}^{3} & 0 & 0 \\
k_{x}^{3} & -\nu \hat{k}_{z} & 0 & 0 \\
0 & 0 & -\nu \hat{k}_{z} & -k_{x}^{3} \\
0 & 0 & -k_{x}^{3} & \nu \hat{k}_{z}
\end{array}\right)+u(z) \mathbb{1}_{4},
$$

where $u(z)=\theta(z+L / 2)(1-\theta(z-L / 2))(\theta$ is the Heaviside function) and for simplicity we considered only incident momenta $k_{x}$ and $k_{z}$. For general combinations of $k_{x}, k_{y}$ and anisotropy parameter $\lambda$ one may apply a rotation to bring the Hamiltonian to this form. Therefore nothing insightful is lost from the discussion using this simplification (see the appendix Appendix $\mathrm{C}$ for details). One finds that the energies in the different regions are given by

$$
\epsilon_{i}=\sqrt{\nu k_{z}^{2}+k_{x}^{6}}+u_{i}
$$

which for the region $z<-L / 2$ is plotted in Fig. 18 for visualization.

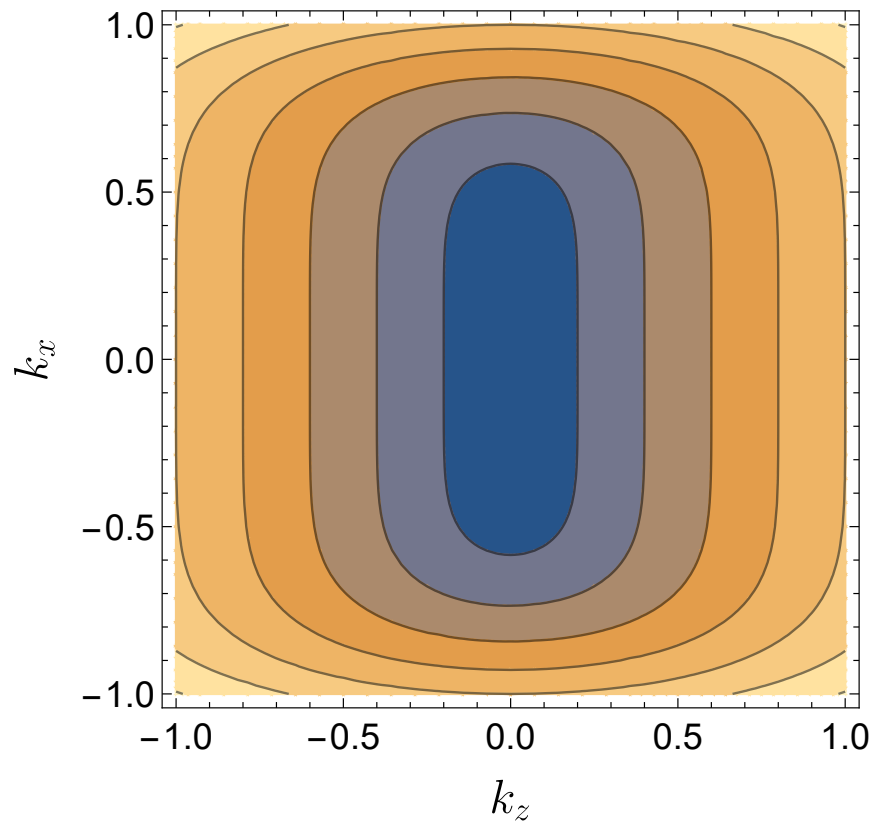

(a) Contour plot of the positive energy band

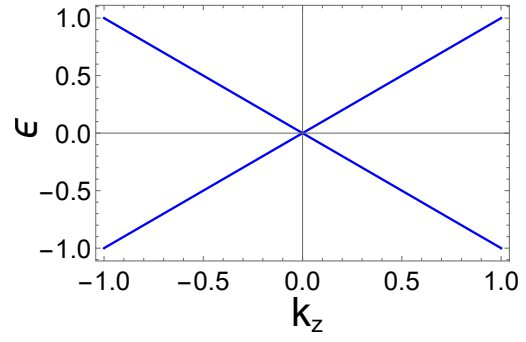

(b) Slice at $k_{x}=0$

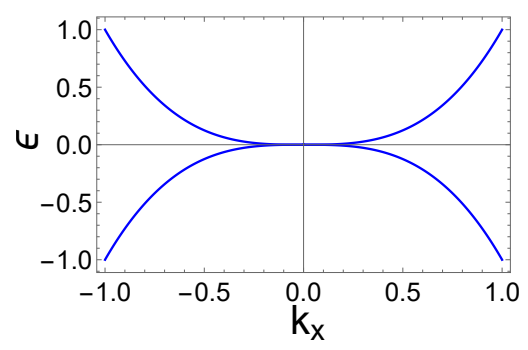

(c) Slice at $k_{z}=0$

Figure 18: (color online) Energy dispersion in the region $z<-L / 2$ for $\nu=1$.

We find that similarly to the case of graphene [6] the scattering problem can be solved by only matching wavefunctions since the barrier is in the linearly dispersive direction. As a result one finds the following transmission amplitudes

$$
T_{1,2}=\frac{1}{\cos ^{2}\left(L q_{z, i}\right)+\frac{\sin ^{2}\left(L q_{z, i}\right)\left(u^{2}-\nu^{2}\left(k_{z, i}^{2}+q_{z, i}^{2}\right)\right)^{2}}{4 k_{z, i}^{2} \nu^{4} q_{z, i}^{2}}},
$$

where we used the definitions $k_{x}=k \sin (\theta), k_{z, i}=k \cos (\theta), \epsilon=\sqrt{\nu k_{z}^{2}+k_{x}^{6}}$ and $q_{z, i}=\frac{1}{\nu} \sqrt{(\epsilon-u)^{2}-k_{x}^{6}}$. This result may be expanded for small $\theta$ to gain further insight. One then finds that

$$
T_{1,2} \approx 1-\frac{k^{4} u^{2} \sin ^{2}\left(\frac{L}{\nu}|u-k \nu|\right)}{\nu^{2}(u-k \nu)^{2}} \theta^{6} .
$$


This result tells us that there is Klein tunneling at small angles and that it is stronger than in the case of graphene and Weyl semi-metals where $T-1 \propto \theta^{2}$. That is for the cubic Dirac semi-metal the Klein tunneling happens over a larger range of angles. A plot of the transmission coefficient as a function of the incident angle $\theta$ can be found in Fig. 19.

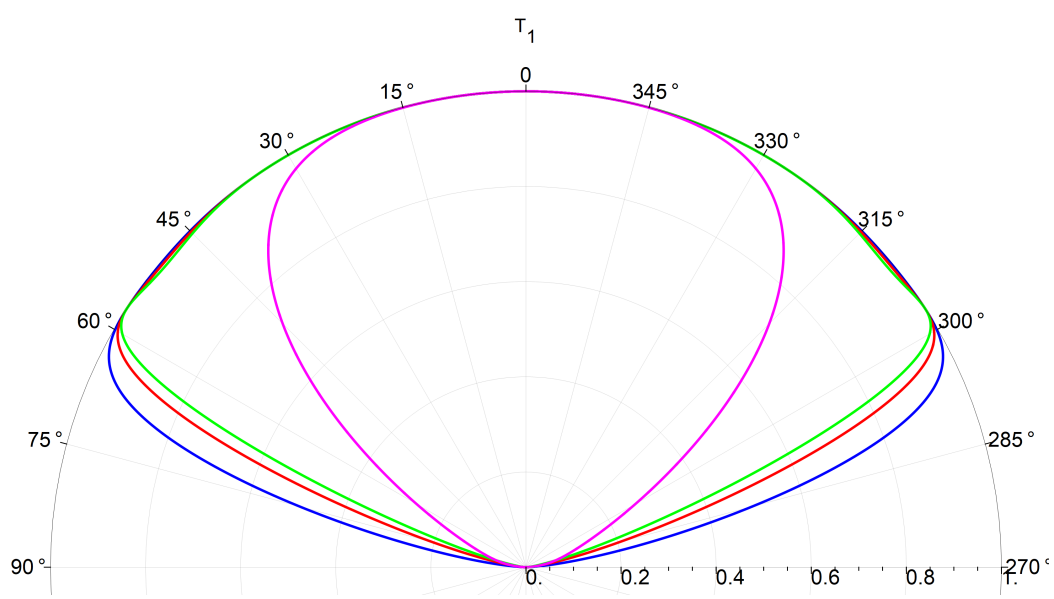

Figure 19: (color online) The transmission coefficient $T_{1}$ for an incident wavevector amplitude $k=1$ and the barrier height $u_{0}=4$ as a function of the incident angle $\theta$ for various values of barrier thickness $L=1$ (blue line), $L=2$ (red line), $L=3$ (green line), $L=3.5$ (magnetia line).

We find observe that $T_{1}$ is very close to unity in a larger angle range $-60^{\circ}<\theta<60^{\circ}$.

One should note that this result can be generalized to arbitrary order Dirac semi-metals as follows

$$
T_{1,2} \approx 1-\frac{\left[u^{2} k^{2(n-1)} \sin ^{2}(L(u-k \nu))\right]}{\nu^{2}(u-k \nu)^{2}} \theta^{2 n}
$$

Therefore Dirac semi-metals of increasing dispersion order (n) have increasingly strong Klein tunneling.

\section{Conclusion}

In this paper we have investigated the tunneling properties of electrons through a potential barrier in semimetals described by a cubically dispersed Dirac equation. The cubic Dirac equation was recently predicted based on space-group symmetry considerations and later found to be realizable in a real solid state system, the quasi-onedimensional transition-metal molybdenum monochalcogenide compound, However, our present work represents the first attempt, to our knowledge, that investigates the theoretical consequences of such a cubically dispersed Dirac system.

Since the considered cubic Dirac equation has many interesting properties such as a linear dispersion in the $z$-direction, cubic dispersion and rotational symmetry or lack of it in the $x-y$ plane, we have designed different situations where each of these effects is isolated and investigated separately. Finally have combined all effects together at a later stage to see if they conspire to create a physical effect that does not occur in isolated situations.

First, we considered the very thin layer limit where the linear dispersion along the $z$-direction is ignored but we maintained the rotational symmetry of the energy dispersion in the $x-y$ plane. In this case, we found that the system behaves similarly to single layer graphene or linearly dispersive Dirac semi-metals. In particular, we found that Klein tunneling at normal incidence persists and, much like in these materials, for small angles behaves as $T-1 \propto \theta^{2}$. However, at oblique incidence on the square barrier we observed the appearance of Fabry-Pérot resonances.

Second, we considered the situation with a non-zero momentum eigenvalue $k_{z}$ which then acts as an effective mass term in the Hamiltonian. In this situation, as expected, we observed a suppression of Klein tunneling except at specific resonant values.

Third, we considered both previous cases but with the inclusion of an anisotropy parameter $\lambda$ that breaks rotational symmetry. In this regime we found that for different incident angles $\theta$ and anistotropy parameter $\lambda$ we were able to get different numbers of real valued wavevector components $k_{x}$. Real valued solutions for $k_{x}$ are the most important solutions since they represent the propagating modes that can be detected by a distant detector. 
This allowed us to draw a phase diagram (Fig. 12) that exposes clearly how many modes can be detected by a distant detector as we vary the incidence angle and anisotropy parameter.

Finally, we considered a potential barrier in the linearly dispersive $z$-direction and observed that Klein tunneling occurs over a larger range of angles as compared to graphene, that is for small angles $T-1 \propto \theta^{6}$.

In summary we demonstrated that cubic Dirac semi-metals host a wealth of interesting tunneling phenomena and that our present theoretical investigation might represent just a tip of an iceberg. We hope, however, that our work paves the way for additional research into transport properties of this phenomenologically rich exotic semi-metal.

\section{Acknowledgements}

We acknowledge the support of King Fahd University of Petroelum and Minerals under Research group project "Transport and semi-classical properties of semi-metals", RGxxxx.

\section{References}

[1] K. S. Novoselov, A. K. Geim, S. V. Morozov, D. Jiang, Y. Zhang, S. V. Dubonos, I. V. Grigorieva, A. A. Firsov, Electric field effect in atomically thin carbon films, science 306 (5696) (2004) 666-669.

[2] A. H. Castro Neto, F. Guinea, N. M. R. Peres, K. S. Novoselov, A. K. Geim, The electronic properties of graphene, Reviews of Modern Physics 81 (1) (2009) 109-162. doi:10.1103/revmodphys.81.109. URL http://dx.doi.org/10.1103/RevModPhys.81.109

[3] A. K. Geim, Graphene: Status and prospects, Science 324 (5934) (2009) 1530-1534. doi:10.1126/science. 1158877.

URL http://dx.doi.org/10.1126/science.1158877

[4] Y. Zhang, Y.-W. Tan, H. L. Stormer, P. Kim, Experimental observation of the quantum hall effect and berry's phase in graphene, nature 438 (7065) (2005) 201-204.

[5] K. Chung, C.-H. Lee, G.-C. Yi, Transferable gan layers grown on zno-coated graphene layers for optoelectronic devices, Science 330 (6004) (2010) 655-657.

[6] M. I. Katsnelson, K. S. Novoselov, A. K. Geim, Chiral tunnelling and the klein paradox in graphene, Nature Physics 2 (9) (2006) 620-625. doi:10.1038/nphys384.

URL http://dx.doi.org/10.1038/nphys384

[7] M. Vogl, O. Pankratov, S. Shallcross, Semiclassics for matrix hamiltonians: The gutzwiller trace formula with applications to graphene-type systems, Physical Review B 96 (3) (2017) 035442.

[8] M. I. Katsnelson, M. I. Katsnelson, Graphene: carbon in two dimensions, Cambridge university press, 2012.

[9] R. Logemann, K. Reijnders, T. Tudorovskiy, M. Katsnelson, S. Yuan, Modeling klein tunneling and caustics of electron waves in graphene, Physical Review B 91 (4) (2015) 045420.

[10] A. F. Young, C. R. Dean, L. Wang, H. Ren, P. Cadden-Zimansky, K. Watanabe, T. Taniguchi, J. Hone, K. L. Shepard, P. Kim, Spin and valley quantum hall ferromagnetism in graphene, Nature Physics 8 (7) (2012) $550-556$.

[11] N. Klier, S. Shallcross, S. Sharma, O. Pankratov, Ruderman-kittel-kasuya-yosida interaction at finite temperature: Graphene and bilayer graphene, Physical Review B 92 (20) (2015) 205414.

[12] R. Bistritzer, A. H. MacDonald, Electronic cooling in graphene, Physical Review Letters 102 (20) (2009) 206410.

[13] K. Nomura, A. H. MacDonald, Quantum hall ferromagnetism in graphene, Physical review letters 96 (25) (2006) 256602.

[14] A. L. Walter, A. Bostwick, K.-J. Jeon, F. Speck, M. Ostler, T. Seyller, L. Moreschini, Y. J. Chang, M. Polini, R. Asgari, et al., Effective screening and the plasmaron bands in graphene, Physical Review B 84 (8) (2011) 085410. 
[15] D. Xiao, W. Yao, Q. Niu, Valley-contrasting physics in graphene: magnetic moment and topological transport, Physical Review Letters 99 (23) (2007) 236809.

[16] P. Carmier, D. Ullmo, Berry phase in graphene: Semiclassical perspective, Physical Review B 77 (24) (2008) 245413.

[17] M. Rodriguez-Vega, J. Fischer, S. D. Sarma, E. Rossi, Ground state of graphene heterostructures in the presence of random charged impurities, Physical Review B 90 (3) (2014) 035406.

[18] C.-P. Lu, M. Rodriguez-Vega, G. Li, A. Luican-Mayer, K. Watanabe, T. Taniguchi, E. Rossi, E. Y. Andrei, Local and global screening properties of graphene revealed through landau level spectroscopy, arXiv preprint arXiv:1504.07540 (2015).

[19] M. Rodriguez-Vega, G. Schwiete, J. Sinova, E. Rossi, Giant edelstein effect in topological-insulator-graphene heterostructures, Physical Review B 96 (23) (2017) 235419.

[20] A. Kormanyos, P. Rakyta, L. Oroszlany, J. Cserti, Bound states in inhomogeneous magnetic field in graphene: Semiclassical approach, Physical Review B 78 (4) (2008) 045430.

[21] K. Reijnders, D. Minenkov, M. Katsnelson, S. Y. Dobrokhotov, Electronic optics in graphene in the semiclassical approximation, Annals of Physics 397 (2018) 65-135.

[22] M. Vogl, M. Rodriguez-Vega, G. A. Fiete, Effective floquet hamiltonians for periodically driven twisted bilayer graphene, Physical Review B 101 (23) (2020) 235411.

[23] P. M. Perez-Piskunow, G. Usaj, C. A. Balseiro, L. F. Torres, Floquet chiral edge states in graphene, Physical Review B 89 (12) (2014) 121401.

[24] G. Usaj, P. M. Perez-Piskunow, L. F. Torres, C. A. Balseiro, Irradiated graphene as a tunable floquet topological insulator, Physical Review B 90 (11) (2014) 115423.

[25] H. L. Calvo, H. M. Pastawski, S. Roche, L. E. F. Torres, Tuning laser-induced band gaps in graphene, Applied Physics Letters 98 (23) (2011) 232103.

[26] L. E. F. Torres, S. Roche, J.-C. Charlier, Introduction to graphene-based nanomaterials: from electronic structure to quantum transport, Cambridge University Press, 2020.

[27] J. M. Pereira Jr, P. Vasilopoulos, F. Peeters, Graphene-based resonant-tunneling structures, Applied physics letters 90 (13) (2007) 132122.

[28] Ö. F. Dayi, A. Jellal, A noncommutative space approach to confined dirac fermions in graphene, Journal of Mathematical physics 51 (6) (2010) 063522.

[29] A. Jellal, Measuring space deformation via graphene under constraints, Physics Letters A 385 (2021) 126964.

[30] M. Mekkaoui, A. Jellal, H. Bahlouli, Fano resonances in gapped graphene subject to an oscillating potential barrier and magnetic field, Physica E: Low-dimensional Systems and Nanostructures (2020) 114502.

[31] H. M. Abdullah, M. Van der Donck, H. Bahlouli, F. Peeters, B. Van Duppen, Graphene quantum blisters: A tunable system to confine charge carriers, Applied Physics Letters 112 (21) (2018) 213101.

[32] H. M. Abdullah, H. Bahlouli, F. Peeters, B. Van Duppen, Confined states in graphene quantum blisters, Journal of Physics: Condensed Matter 30 (38) (2018) 385301.

[33] G. Berdiyorov, H. Bahlouli, F. Peeters, Effect of substitutional impurities on the electronic transport properties of graphene, Physica E: Low-dimensional Systems and Nanostructures 84 (2016) 22-26.

[34] Y. Zahidi, I. Redouani, A. Jellal, H. Bahlouli, Magnetic field effect on strained graphene junctions, Physica E: Low-dimensional Systems and Nanostructures 115 (2020) 113672.

[35] B. Partoens, F. Peeters, From graphene to graphite: Electronic structure around the k point, Physical Review B 74 (7) (2006) 075404. 
[36] F. Giustino, J. H. Lee, F. Trier, M. Bibes, S. M. Winter, R. Valentí, Y.-W. Son, L. Taillefer, C. Heil, A. I. Figueroa, B. Plaçais, Q. Wu, O. V. Yazyev, E. P. A. M. Bakkers, J. Nygård, P. Forn-Díaz, S. D. Franceschi, J. W. McIver, L. E. F. F. Torres, T. Low, A. Kumar, R. Galceran, S. O. Valenzuela, M. V. Costache, A. Manchon, E.-A. Kim, G. R. Schleder, A. Fazzio, S. Roche, The 2021 quantum materials roadmap, Journal of Physics: Materials 3 (4) (2021) 042006. doi:10.1088/2515-7639/abb74e.

URL https://doi.org/10.1088/2515-7639/abb74e

[37] J. R. Schaibley, H. Yu, G. Clark, P. Rivera, J. S. Ross, K. L. Seyler, W. Yao, X. Xu, Valleytronics in 2d materials, Nature Reviews Materials 1 (11) (2016) 1-15.

[38] K. Novoselov, o. A. Mishchenko, o. A. Carvalho, A. C. Neto, 2d materials and van der waals heterostructures, Science 353 (6298) (2016).

[39] P. Avouris, T. F. Heinz, T. Low, 2D Materials, Cambridge University Press, 2017.

[40] M. Gibertini, M. Koperski, A. Morpurgo, K. Novoselov, Magnetic 2d materials and heterostructures, Nature nanotechnology 14 (5) (2019) 408-419.

[41] R. Mas-Balleste, C. Gomez-Navarro, J. Gomez-Herrero, F. Zamora, 2d materials: to graphene and beyond, Nanoscale 3 (1) (2011) 20-30.

[42] Y. K. Ryu, R. Frisenda, A. Castellanos-Gomez, Superlattices based on van der waals 2d materials, Chemical Communications 55 (77) (2019) 11498-11510.

[43] R. M. Wallace, In-situ studies on 2d materials, Ecs Transactions 64 (9) (2014) 109.

[44] I. Choudhuri, P. Bhauriyal, B. Pathak, Recent advances in graphene-like 2d materials for spintronics applications, Chemistry of Materials 31 (20) (2019) 8260-8285.

[45] P. Vogt, P. De Padova, C. Quaresima, J. Avila, E. Frantzeskakis, M. C. Asensio, A. Resta, B. Ealet, G. Le Lay, Silicene: compelling experimental evidence for graphenelike two-dimensional silicon, Physical review letters $108(15)$ (2012) 155501.

[46] Z. Ni, Q. Liu, K. Tang, J. Zheng, J. Zhou, R. Qin, Z. Gao, D. Yu, J. Lu, Tunable bandgap in silicene and germanene, Nano letters 12 (1) (2012) 113-118.

[47] A. Kara, H. Enriquez, A. P. Seitsonen, L. L. Y. Voon, S. Vizzini, B. Aufray, H. Oughaddou, A review on silicene - new candidate for electronics, Surface science reports 67 (1) (2012) 1-18.

[48] A. Kara, C. Léandri, M. Dávila, P. De Padova, B. Ealet, H. Oughaddou, B. Aufray, G. Le Lay, Physics of silicene stripes, Journal of superconductivity and novel magnetism 22 (3) (2009) 259-263.

[49] B. Lalmi, H. Oughaddou, H. Enriquez, A. Kara, S. Vizzini, B. Ealet, B. Aufray, Epitaxial growth of a silicene sheet, Applied Physics Letters 97 (22) (2010) 223109.

[50] D. Xiao, G.-B. Liu, W. Feng, X. Xu, W. Yao, Coupled spin and valley physics in monolayers ofmos2and other group-vi dichalcogenides, Physical Review Letters 108 (19) (May 2012). doi:10.1103/physrevlett.108. 196802.

URL http://dx.doi.org/10.1103/PhysRevLett.108.196802

[51] G.-B. Liu, W.-Y. Shan, Y. Yao, W. Yao, D. Xiao, Three-band tight-binding model for monolayers of group-vib transition metal dichalcogenides, Phys. Rev. B 88 (2013) 085433. doi:10.1103/PhysRevB.88.085433. URL https://link.aps.org/doi/10.1103/PhysRevB.88.085433

[52] S. Manzeli, D. Ovchinnikov, D. Pasquier, O. V. Yazyev, A. Kis, 2d transition metal dichalcogenides, Nature Reviews Materials 2 (8) (2017) 17033.

[53] Q. H. Wang, K. Kalantar-Zadeh, A. Kis, J. N. Coleman, M. S. Strano, Electronics and optoelectronics of two-dimensional transition metal dichalcogenides, Nature nanotechnology 7 (11) (2012) 699-712.

[54] H.-L. Liu, C.-C. Shen, S.-H. Su, C.-L. Hsu, M.-Y. Li, L.-J. Li, Optical properties of monolayer transition metal dichalcogenides probed by spectroscopic ellipsometry, Applied Physics Letters 105 (20) (2014) 201905. 
[55] R. Friend, A. Yoffe, Electronic properties of intercalation complexes of the transition metal dichalcogenides, Advances in Physics 36 (1) (1987) 1-94.

[56] D. Voiry, A. Mohite, M. Chhowalla, Phase engineering of transition metal dichalcogenides, Chemical Society Reviews 44 (9) (2015) 2702-2712.

[57] M. Chhowalla, Z. Liu, H. Zhang, Two-dimensional transition metal dichalcogenide (tmd) nanosheets, Chemical Society Reviews 44 (9) (2015) 2584-2586.

[58] D. Azhikodan, T. Nautiyal, S. Shallcross, S. Sharma, An anomalous interlayer exciton in mos 2, Scientific reports 6 (1) (2016) 1-6.

[59] H. Min, A. H. MacDonald, Electronic structure of multilayer graphene, Progress of Theoretical Physics Supplement 176 (2008) 227-252.

[60] F. Wu, A. MacDonald, I. Martin, Theory of phonon-mediated superconductivity in twisted bilayer graphene, Physical Review Letters 121 (25) (Dec 2018). doi:10.1103/physrevlett.121.257001.

URL http://dx.doi.org/10.1103/PhysRevLett.121.257001

[61] R. Bistritzer, A. H. MacDonald, Moiré bands in twisted double-layer graphene, Proceedings of the National Academy of Sciences 108 (30) (2011) 12233-12237. arXiv:https://www.pnas.org/content/108/30/12233. full.pdf, doi:10.1073/pnas.1108174108.

URL https://www.pnas.org/content/108/30/12233

[62] Y. Cao, V. Fatemi, S. Fang, K. Watanabe, T. Taniguchi, E. Kaxiras, P. Jarillo-Herrero, Unconventional superconductivity in magic-angle graphene superlattices, Nature 556 (7699) (2018) 43-50. doi:10.1038/ nature26160.

URL https://doi.org/10.1038/nature26160

[63] E. Codecido, Q. Wang, R. Koester, S. Che, H. Tian, R. Lv, S. Tran, K. Watanabe, T. Taniguchi, F. Zhang, M. Bockrath, C. N. Lau, Correlated insulating and superconducting states in twisted bilayer graphene below the magic angle, Science Advances 5 (9) (2019). doi:10.1126/sciadv. aaw9770.

URL https://advances.sciencemag.org/content/5/9/eaaw9770

[64] M. Yankowitz, S. Chen, H. Polshyn, Y. Zhang, K. Watanabe, T. Taniguchi, D. Graf, A. F. Young, C. R. Dean, Tuning superconductivity in twisted bilayer graphene, Science 363 (6431) (2019) 1059-1064. doi: 10.1126/science. aav1910. URL https://science.sciencemag.org/content/363/6431/1059

[65] D. V. Chichinadze, L. Classen, A. V. Chubukov, Nematic superconductivity in twisted bilayer graphene, arXiv e-prints (2019) arXiv:1910.07379arXiv:1910.07379.

[66] M. Fleischmann, R. Gupta, S. Sharma, S. Shallcross, Moiré quantum well states in tiny angle two dimensional semi-conductors, arXiv e-prints (2019) arXiv:1901.04679arXiv:1901.04679.

[67] F. Wu, T. Lovorn, E. Tutuc, I. Martin, A. MacDonald, Topological insulators in twisted transition metal dichalcogenide homobilayers, Physical Review Letters 122 (8) (Feb 2019). doi:10.1103/physrevlett.122. 086402.

URL http://dx.doi.org/10.1103/PhysRevLett.122.086402

[68] D. Zhai, W. Yao, Theory of tunable flux lattices in the homobilayer moiré of twisted and uniformly strained transition metal dichalcogenides (2020). arXiv:2006.02306.

[69] Y. Zhang, Z. Zhan, F. Guinea, J. A. Silva-Guillen, S. Yuan, Tuning band gaps in twisted bilayer $\operatorname{mos}_{2}(2020)$. arXiv:2005.13879.

[70] Z. Zhan, Y. Zhang, G. Yu, F. G. J. A. Silva-Guillen, S. Yuan, Multi-ultraflatbands tunability and effect of spin-orbit coupling in twisted bilayer transition metal dichalcogenides (2020). arXiv:2005.13868.

[71] S. Venkateswarlu, A. Honecker, G. T. de Laissardière, Electronic localization in twisted bilayer mos 2 with small rotation angle (2020). arXiv:2005.13054. 
[72] M. Chen, X. Lin, T. Dinh, Z. Zheng, J. Shen, Q. Ma, H. Chen, P. Jarillo-Herrero, S. Dai, Configure polaritons in twisted $\alpha$-moo3 (2020). arXiv:2004.14588.

[73] P. San-Jose, J. González, F. Guinea, Non-abelian gauge potentials in graphene bilayers, Physical Review Letters 108 (21) (May 2012). doi:10.1103/physrevlett.108.216802.

URL http://dx.doi.org/10.1103/PhysRevLett.108.216802

[74] Y. Zhang, K. Jiang, Z. Wang, F. Zhang, Correlated insulating phases of twisted bilayer graphene at commensurate filling fractions: a hartree-fock study, Physical Review B 102 (3) (2020) 035136.

[75] F. Xie, Z. Song, B. Lian, B. A. Bernevig, Topology-bounded superfluid weight in twisted bilayer graphene, Physical review letters 124 (16) (2020) 167002.

[76] F. Wu, S. D. Sarma, Collective excitations of quantum anomalous hall ferromagnets in twisted bilayer graphene, Physical review letters 124 (4) (2020) 046403.

[77] S. Shallcross, S. Sharma, O. A. Pankratov, Quantum interference at the twist boundary in graphene, Physical review letters 101 (5) (2008) 056803.

[78] M. Vogl, M. Rodriguez-Vega, G. A. Fiete, Floquet engineering of interlayer couplings: Tuning the magic angle of twisted bilayer graphene at the exit of a waveguide, Physical Review B 101 (24) (2020) 241408.

[79] F. Rost, R. Gupta, M. Fleischmann, D. Weckbecker, N. Ray, J. Olivares, M. Vogl, S. Sharma, O. Pankratov, S. Shallcross, Nonperturbative theory of effective hamiltonians for deformations in two-dimensional materials: Moiré systems and dislocations, Physical Review B 100 (3) (2019) 035101.

[80] J. Jung, F. Zhang, Z. Qiao, A. H. MacDonald, Valley-hall kink and edge states in multilayer graphene, Physical Review B 84 (7) (2011) 075418.

[81] H. Min, A. H. MacDonald, Chiral decomposition in the electronic structure of graphene multilayers, Physical Review B 77 (15) (2008) 155416.

[82] S.-E. Zhu, S. Yuan, G. Janssen, Optical transmittance of multilayer graphene, EPL (Europhysics Letters) 108 (1) (2014) 17007.

[83] H. M. Abdullah, M. Al Ezzi, H. Bahlouli, Electronic transport and klein tunneling in gapped aa-stacked bilayer graphene, Journal of Applied Physics 124 (20) (2018) 204303.

[84] H. Abdullah, M. Zarenia, H. Bahlouli, F. Peeters, B. Van Duppen, Gate tunable layer selectivity of transport in bilayer graphene nanostructures, EPL (Europhysics Letters) 113 (1) (2016) 17006.

[85] Z. Zhu, S. Carr, D. Massatt, M. Luskin, E. Kaxiras, Twisted trilayer graphene: a precisely tunable platform for correlated electrons, Physical review letters 125 (11) (2020) 116404.

[86] Z. Ma, S. Li, Y.-W. Zheng, M.-M. Xiao, H. Jiang, J.-H. Gao, X. Xie, Topological flat bands in twisted trilayer graphene, Science Bulletin (2020).

[87] Y. Shi, S. Xu, M. M. A. Ezzi, N. Balakrishnan, A. Garcia-Ruiz, B. Tsim, C. Mullan, J. Barrier, N. Xin, B. A. Piot, et al., Tunable van hove singularities and correlated states in twisted trilayer graphene, arXiv preprint arXiv:2004.12414 (2020).

[88] W.-J. Zuo, J.-B. Qiao, D.-L. Ma, L.-J. Yin, G. Sun, J.-Y. Zhang, L.-Y. Guan, L. He, Scanning tunneling microscopy and spectroscopy of twisted trilayer graphene, Physical Review B 97 (3) (2018) 035440.

[89] B. Amorim, E. V. Castro, Electronic spectral properties of incommensurate twisted trilayer graphene, arXiv preprint arXiv:1807.11909 (2018).

[90] A. Lopez-Bezanilla, J. Lado, Electrical band flattening, valley flux, and superconductivity in twisted trilayer graphene, Physical Review Research 2 (3) (2020) 033357.

[91] C. Lei, L. Linhart, W. Qin, F. Libisch, A. H. MacDonald, Mirror symmetry breaking and stacking-shift dependence in twisted trilayer graphene, arXiv preprint arXiv:2010.05787 (2020). 
[92] X. Li, F. Wu, A. H. MacDonald, Electronic structure of single-twist trilayer graphene, arXiv preprint arXiv:1907.12338 (2019).

[93] C. Mora, N. Regnault, B. A. Bernevig, Flatbands and perfect metal in trilayer moiré graphene, Physical review letters 123 (2) (2019) 026402.

[94] V. Vitale, K. Atalar, A. A. Mostofi, J. Lischner, Flat band properties of twisted transition metal dichalcogenide homo- and heterobilayers of $\operatorname{mos}_{2}, \mathrm{mose}_{2}, \mathrm{ws}_{2}$ and wse 2 (2021). arXiv:2102.03259.

[95] N. Armitage, E. Mele, A. Vishwanath, Weyl and dirac semimetals in three-dimensional solids, Reviews of Modern Physics 90 (1) (Jan 2018). doi:10.1103/revmodphys.90.015001.

URL http://dx.doi.org/10.1103/RevModPhys.90.015001

[96] B. Yan, C. Felser, Topological materials: Weyl semimetals, Annual Review of Condensed Matter Physics 8 (1) (2017) 337-354. arXiv:https://doi.org/10.1146/annurev-conmatphys-031016-025458, doi:10. 1146/annurev-conmatphys-031016-025458.

URL https://doi.org/10.1146/annurev-conmatphys-031016-025458

[97] S. Wang, B.-C. Lin, A.-Q. Wang, D.-P. Yu, Z.-M. Liao, Quantum transport in dirac and weyl semimetals: a review, Advances in Physics: X 2 (3) (2017) 518-544.

[98] Q. Liu, A. Zunger, Predicted realization of cubic dirac fermion in quasi-one-dimensional transition-metal monochalcogenides, Phys. Rev. X 7 (2017) 021019. doi:10.1103/PhysRevX.7.021019.

URL https://link.aps.org/doi/10.1103/PhysRevX.7.021019

[99] A. A. Zyuzin, R. P. Tiwari, Intrinsic anomalous hall effect in type-ii weyl semimetals, JETP letters 103 (11) (2016) 717-722.

[100] J. Jiang, Z. Liu, Y. Sun, H. Yang, C. Rajamathi, Y. Qi, L. Yang, C. Chen, H. Peng, C. Hwang, et al., Signature of type-ii weyl semimetal phase in mote 2, Nature communications 8 (1) (2017) 1-6.

[101] Z. Wang, D. Gresch, A. A. Soluyanov, W. Xie, S. Kushwaha, X. Dai, M. Troyer, R. J. Cava, B. A. Bernevig, Mote 2: a type-ii weyl topological metal, Physical review letters 117 (5) (2016) 056805.

[102] H. Weng, C. Fang, Z. Fang, B. A. Bernevig, X. Dai, Weyl semimetal phase in noncentrosymmetric transitionmetal monophosphides, Physical Review X 5 (1) (2015) 011029.

[103] S.-Y. Xu, I. Belopolski, N. Alidoust, M. Neupane, G. Bian, C. Zhang, R. Sankar, G. Chang, Z. Yuan, C.C. Lee, et al., Discovery of a weyl fermion semimetal and topological fermi arcs, Science 349 (6248) (2015) 613-617.

[104] A. A. Soluyanov, D. Gresch, Z. Wang, Q. Wu, M. Troyer, X. Dai, B. A. Bernevig, Type-ii weyl semimetals, Nature 527 (7579) (2015) 495-498.

[105] S.-M. Huang, S.-Y. Xu, I. Belopolski, C.-C. Lee, G. Chang, B. Wang, N. Alidoust, G. Bian, M. Neupane, C. Zhang, et al., A weyl fermion semimetal with surface fermi arcs in the transition metal monopnictide taas class, Nature communications 6 (1) (2015) 1-6.

[106] X. Huang, L. Zhao, Y. Long, P. Wang, D. Chen, Z. Yang, H. Liang, M. Xue, H. Weng, Z. Fang, et al., Observation of the chiral-anomaly-induced negative magnetoresistance in $3 \mathrm{~d}$ weyl semimetal taas, Physical Review X 5 (3) (2015) 031023.

[107] A. Zyuzin, S. Wu, A. Burkov, Weyl semimetal with broken time reversal and inversion symmetries, Physical Review B 85 (16) (2012) 165110.

[108] R. Lundgren, P. Laurell, G. A. Fiete, Thermoelectric properties of weyl and dirac semimetals, Physical Review B 90 (16) (2014) 165115.

[109] R. Lundgren, G. A. Fiete, Electronic cooling in weyl and dirac semimetals, Physical Review B 92 (12) (2015) 125139.

[110] D. Bulmash, C.-X. Liu, X.-L. Qi, Prediction of a weyl semimetal in hg 1- x- y cd x mn y te, Physical Review B 89 (8) (2014) 081106. 
[111] J.-H. Jiang, Tunable topological weyl semimetal from simple-cubic lattices with staggered fluxes, Physical Review A 85 (3) (2012) 033640.

[112] A. Burkov, L. Balents, Weyl semimetal in a topological insulator multilayer, Physical review letters 107 (12) (2011) 127205.

[113] H.-X. Wang, Z.-K. Lin, B. Jiang, G.-Y. Guo, J.-H. Jiang, Higher-order weyl semimetals, Physical Review Letters 125 (14) (2020) 146401.

[114] S. A. A. Ghorashi, T. Li, T. L. Hughes, Higher-order weyl semimetals, Physical Review Letters 125 (26) (2020) 266804.

[115] R. Wang, B. Wang, R. Shen, L. Sheng, D. Xing, Floquet weyl semimetal induced by off-resonant light, EPL (Europhysics Letters) 105 (1) (2014) 17004.

[116] X.-X. Zhang, T. T. Ong, N. Nagaosa, Theory of photoinduced floquet weyl semimetal phases, Physical Review B 94 (23) (2016) 235137.

[117] H. Hübener, M. A. Sentef, U. De Giovannini, A. F. Kemper, A. Rubio, Creating stable floquet-weyl semimetals by laser-driving of 3d dirac materials, Nature communications 8 (1) (2017) 1-8.

[118] Q. Chen, G. A. Fiete, Thermoelectric transport in double-weyl semimetals, Physical Review B 93 (15) (2016) 155125.

[119] B. Lv, H. Weng, B. Fu, X. P. Wang, H. Miao, J. Ma, P. Richard, X. Huang, L. Zhao, G. Chen, et al., Experimental discovery of weyl semimetal taas, Physical Review X 5 (3) (2015) 031013.

[120] D. Liu, A. Liang, E. Liu, Q. Xu, Y. Li, C. Chen, D. Pei, W. Shi, S. Mo, P. Dudin, et al., Magnetic weyl semimetal phase in a kagomé crystal, Science 365 (6459) (2019) 1282-1285.

[121] M. Vazifeh, M. Franz, Electromagnetic response of weyl semimetals, Physical review letters 111 (2) (2013) 027201.

[122] M. Alidoust, K. Halterman, Evolution of pair correlation symmetries and supercurrent reversal in tilted weyl semimetals, Physical Review B 101 (3) (Jan 2020). doi:10.1103/physrevb.101.035120.

URL http://dx.doi.org/10.1103/PhysRevB.101.035120

[123] M. Alidoust, Self-biased current, magnetic interference response, and superconducting vortices in tilted weyl semimetals with disorder, Physical Review B 98 (24) (Dec 2018). doi:10.1103/physrevb.98.245418.

URL http://dx.doi.org/10.1103/PhysRevB.98.245418

[124] K. Halterman, M. Alidoust, Waveguide modes in weyl semimetals with tilted dirac cones, Optics Express 27 (25) (2019) 36164. doi:10.1364/oe.27.036164.

URL http://dx.doi.org/10.1364/OE.27.036164

[125] K. Halterman, M. Alidoust, A. Zyuzin, Epsilon-near-zero response and tunable perfect absorption in weyl semimetals, Physical Review B 98 (8) (Aug 2018). doi:10.1103/physrevb.98.085109.

URL http://dx.doi.org/10.1103/PhysRevB.98.085109

[126] M. Alidoust, K. Halterman, A. A. Zyuzin, Superconductivity in type-ii weyl semimetals, Physical Review B 95 (15) (Apr 2017). doi:10.1103/physrevb.95.155124.

URL http://dx.doi.org/10.1103/PhysRevB.95.155124

[127] S.-M. Huang, S.-Y. Xu, I. Belopolski, C.-C. Lee, G. Chang, T.-R. Chang, B. Wang, N. Alidoust, G. Bian, M. Neupane, et al., New type of weyl semimetal with quadratic double weyl fermions, Proceedings of the National Academy of Sciences 113 (5) (2016) 1180-1185.

[128] X. Wan, A. M. Turner, A. Vishwanath, S. Y. Savrasov, Topological semimetal and fermi-arc surface states in the electronic structure of pyrochlore iridates, Physical Review B 83 (20) (2011) 205101.

[129] G. Xu, H. Weng, Z. Wang, X. Dai, Z. Fang, Chern semimetal and the quantized anomalous hall effect in hgcr 2 se 4, Physical review letters 107 (18) (2011) 186806. 
[130] C. Fang, M. J. Gilbert, X. Dai, B. A. Bernevig, Multi-weyl topological semimetals stabilized by point group symmetry, Physical review letters 108 (26) (2012) 266802.

[131] J. Xiong, S. K. Kushwaha, T. Liang, J. W. Krizan, M. Hirschberger, W. Wang, R. J. Cava, N. P. Ong, Evidence for the chiral anomaly in the dirac semimetal na3bi, Science 350 (6259) (2015) 413-416.

[132] Z. Liu, J. Jiang, B. Zhou, Z. Wang, Y. Zhang, H. Weng, D. Prabhakaran, S. K. Mo, H. Peng, P. Dudin, et al., A stable three-dimensional topological dirac semimetal cd 3 as 2, Nature materials 13 (7) (2014) 677-681.

[133] Z. Wang, H. Weng, Q. Wu, X. Dai, Z. Fang, Three-dimensional dirac semimetal and quantum transport in cd 3 as 2, Physical Review B 88 (12) (2013) 125427.

[134] T. Liang, Q. Gibson, M. N. Ali, M. Liu, R. Cava, N. Ong, Ultrahigh mobility and giant magnetoresistance in the dirac semimetal cd 3 as 2, Nature materials 14 (3) (2015) 280-284.

[135] M. Novak, S. Sasaki, K. Segawa, Y. Ando, Large linear magnetoresistance in the dirac semimetal tlbisse, Physical Review B 91 (4) (2015) 041203.

[136] B. J. Wieder, Y. Kim, A. Rappe, C. Kane, Double dirac semimetals in three dimensions, Physical review letters 116 (18) (2016) 186402.

[137] L. He, X. Hong, J. Dong, J. Pan, Z. Zhang, J. Zhang, S. Li, Quantum transport evidence for the threedimensional dirac semimetal phase in cd 3 as 2, Physical review letters 113 (24) (2014) 246402.

[138] S. Jeon, B. B. Zhou, A. Gyenis, B. E. Feldman, I. Kimchi, A. C. Potter, Q. D. Gibson, R. J. Cava, A. Vishwanath, A. Yazdani, Landau quantization and quasiparticle interference in the three-dimensional dirac semimetal cd 3 as 2, Nature materials 13 (9) (2014) 851-856.

[139] C. Weeks, M. Franz, Interaction-driven instabilities of a dirac semimetal, Physical Review B 81 (8) (2010) 085105.

[140] J. Kim, S. S. Baik, S. H. Ryu, Y. Sohn, S. Park, B.-G. Park, J. Denlinger, Y. Yi, H. J. Choi, K. S. Kim, Observation of tunable band gap and anisotropic dirac semimetal state in black phosphorus, Science 349 (6249) (2015) 723-726.

[141] P. Li, C. Zhang, J. Zhang, Y. Wen, X. Zhang, Giant planar hall effect in the dirac semimetal zrt e 5- $\delta$, Physical Review B 98 (12) (2018) 121108.

[142] T.-R. Chang, S.-Y. Xu, D. S. Sanchez, W.-F. Tsai, S.-M. Huang, G. Chang, C.-H. Hsu, G. Bian, I. Belopolski, Z.-M. Yu, et al., Type-ii symmetry-protected topological dirac semimetals, Physical review letters 119 (2) (2017) 026404.

[143] A. L. Szabó, B. Roy, Dirty higher-order dirac semimetal: Quantum criticality and bulk-boundary correspondence, Physical Review Research 2 (4) (2020) 043197.

[144] B. J. Wieder, Z. Wang, J. Cano, X. Dai, L. M. Schoop, B. Bradlyn, B. A. Bernevig, Strong and fragile topological dirac semimetals with higher-order fermi arcs, Nature communications 11 (1) (2020) 1-13.

[145] Z. Wang, Y. Sun, X.-Q. Chen, C. Franchini, G. Xu, H. Weng, X. Dai, Z. Fang, Dirac semimetal and topological phase transitions in a 3 bi (a= na, k, rb), Physical Review B 85 (19) (2012) 195320.

[146] S. M. Young, S. Zaheer, J. C. Teo, C. L. Kane, E. J. Mele, A. M. Rappe, Dirac semimetal in three dimensions, Physical review letters 108 (14) (2012) 140405.

[147] Z. Liu, B. Zhou, Y. Zhang, Z. Wang, H. Weng, D. Prabhakaran, S.-K. Mo, Z. Shen, Z. Fang, X. Dai, et al., Discovery of a three-dimensional topological dirac semimetal, na3bi, Science 343 (6173) (2014) 864-867.

[148] B.-J. Yang, N. Nagaosa, Classification of stable three-dimensional dirac semimetals with nontrivial topology, Nature communications 5 (1) (2014) 1-10.

[149] S. M. Young, C. L. Kane, Dirac semimetals in two dimensions, Physical review letters 115 (12) (2015) 126803.

[150] S. Borisenko, Q. Gibson, D. Evtushinsky, V. Zabolotnyy, B. Büchner, R. J. Cava, Experimental realization of a three-dimensional dirac semimetal, Physical review letters 113 (2) (2014) 027603. 
[151] A. Geim, K. Novoselov, The rise of graphene, Nature Materials 6 (3) (2007) 183-191. doi:10.1038/nmat1849.

[152] C. Fang, H. Weng, X. Dai, Z. Fang, Topological nodal line semimetals, Chinese Physics B 25 (11) (2016) 117106. doi:10.1088/1674-1056/25/11/117106. URL http://dx.doi.org/10.1088/1674-1056/25/11/117106

[153] M. Xiao, L. Ye, C. Qiu, H. He, Z. Liu, S. Fan, Experimental demonstration of acoustic semimetal with topologically charged nodal surface, Science advances 6 (8) (2020) eaav2360.

[154] Y. Qie, J. Liu, S. Wang, Q. Sun, P. Jena, Tetragonal c 24: a topological nodal-surface semimetal with potential as an anode material for sodium ion batteries, Journal of Materials Chemistry A 7 (10) (2019) 5733-5739.

[155] X. Zhang, Z.-M. Yu, Z. Zhu, W. Wu, S.-S. Wang, X.-L. Sheng, S. A. Yang, Nodal loop and nodal surface states in the ti 3 al family of materials, Physical Review B 97 (23) (2018) 235150.

[156] M. Xiao, S. Fan, Topologically charged nodal surface, arXiv preprint arXiv:1709.02363 (2017).

[157] A. Burkov, M. Hook, L. Balents, Topological nodal semimetals, Physical Review B 84 (23) (2011) 235126.

[158] W. Meng, Y. Liu, X. Zhang, X. Dai, G. Liu, A nonsymmorphic-symmetry-protected hourglass weyl node, hybrid weyl node, nodal surface, and dirac nodal line in pd $4 \mathrm{x}(\mathrm{x}=\mathrm{s}, \mathrm{se})$ compounds, Physical Chemistry Chemical Physics 22 (39) (2020) 22399-22407.

[159] J. Wang, Y. Liu, K.-H. Jin, X. Sui, L. Zhang, W. Duan, F. Liu, B. Huang, Pseudo dirac nodal sphere semimetal, Physical Review B 98 (20) (2018) 201112.

[160] E. Emmanouilidou, B. Shen, X. Deng, T.-R. Chang, A. Shi, G. Kotliar, S.-Y. Xu, N. Ni, Magnetotransport properties of the single-crystalline nodal-line semimetal candidates ca $\mathrm{t} x(\mathrm{t}=\mathrm{ag}, \mathrm{cd} ; \mathrm{x}=\mathrm{as}$, ge), Physical Review B 95 (24) (2017) 245113.

[161] Z. Li, W. Wang, P. Zhou, Z. Ma, L. Sun, New type of hybrid nodal line semimetal in be2si, New Journal of Physics 21 (3) (2019) 033018.

[162] H. Huang, J. Liu, D. Vanderbilt, W. Duan, Topological nodal-line semimetals in alkaline-earth stannides, germanides, and silicides, Physical Review B 93 (20) (2016) 201114.

[163] S. Li, Z. Guo, D. Fu, X.-C. Pan, J. Wang, K. Ran, S. Bao, Z. Ma, Z. Cai, R. Wang, et al., Evidence for a dirac nodal-line semimetal in sras3, Science bulletin 63 (9) (2018) 535-541.

[164] R. Yu, Z. Fang, X. Dai, H. Weng, Topological nodal line semimetals predicted from first-principles calculations, Frontiers of Physics 12 (3) (2017) 1-14.

[165] J. He, X. Kong, W. Wang, S.-P. Kou, Type-ii nodal line semimetal, New Journal of Physics 20 (5) (2018) 053019 .

[166] Z.-Z. Li, J. Chen, S. Nie, L. Xu, H. Mizuseki, H. Weng, J.-T. Wang, Orthorhombic carbon oc24: A novel topological nodal line semimetal, Carbon 133 (2018) 39-43.

[167] A. Laha, S. Mardanya, B. Singh, H. Lin, A. Bansil, A. Agarwal, Z. Hossain, Magnetotransport properties of the topological nodal-line semimetal cacdsn, Physical Review B 102 (3) (2020) 035164.

[168] W. Wu, Y. Liu, S. Li, C. Zhong, Z.-M. Yu, X.-L. Sheng, Y. Zhao, S. A. Yang, Nodal surface semimetals: Theory and material realization, Physical Review B 97 (11) (2018) 115125.

[169] X. Zhang, L. Jin, X. Dai, G. Liu, Topological type-ii nodal line semimetal and dirac semimetal state in stable kagome compound mg3bi2, The journal of physical chemistry letters 8 (19) (2017) 4814-4819.

[170] M. M. Hosen, K. Dimitri, I. Belopolski, P. Maldonado, R. Sankar, N. Dhakal, G. Dhakal, T. Cole, P. M. Oppeneer, D. Kaczorowski, et al., Tunability of the topological nodal-line semimetal phase in zrsi x-type materials (x=s, se, te), Physical Review B 95 (16) (2017) 161101.

[171] T.-R. Chang, I. Pletikosic, T. Kong, G. Bian, A. Huang, J. Denlinger, S. K. Kushwaha, B. Sinkovic, H.-T. Jeng, T. Valla, et al., Realization of a type-ii nodal-line semimetal in mg3bi2, Advanced Science 6 (4) (2019) 1800897. 
[172] I. Mandal, A. Sen, Tunneling of multi-weyl semimetals through a potential barrier under the influence of magnetic fields (2021). arXiv:2012.15567.

[173] I. Mandal, Tunneling in fermi systems with quadratic band crossing points, Annals of Physics 419 (2020) 168235. doi:10.1016/j.aop.2020.168235.

URL http://dx.doi.org/10.1016/j.aop.2020.168235

[174] S. Bera, I. Mandal, Floquet scattering of quadratic band-touching semimetals through a time-periodic potential well (2021). arXiv:2103.05335.

[175] I. Mandal, Transmission in pseudospin-1 and pseudospin-3/2 semimetals with linear dispersion through scalar and vector potential barriers, Physics Letters A 384 (27) (2020) 126666. doi:10.1016/j.physleta. 2020. 126666.

URL http://dx.doi.org/10.1016/j.physleta.2020.126666

[176] M. Alidoust, C. Shen, I. Žutić, Cubic spin-orbit coupling and anomalous josephson effect in planar junctions, Physical Review B 103 (6) (Feb 2021). doi:10.1103/physrevb.103.1060503.

URL http://dx.doi.org/10.1103/PhysRevB.103.L060503

[177] J. Wurm, K. Richter, I. Adagideli, Edge effects in graphene nanostructures: From multiple reflection expansion to density of states, Physical Review B 84 (7) (Aug 2011). doi:10.1103/physrevb.84.075468.

URL http://dx.doi.org/10.1103/PhysRevB.84.075468

[178] A. R. Akhmerov, C. W. J. Beenakker, Boundary conditions for dirac fermions on a terminated honeycomb lattice, Physical Review B 77 (8) (Feb 2008). doi:10.1103/physrevb.77.085423.

URL http://dx.doi.org/10.1103/PhysRevB.77.085423

[179] M. V. Berry, R. J. Mondragon, Neutrino billiards: time-reversal symmetry-breaking without magnetic fields, Proceedings of the Royal Society of London. A. Mathematical and Physical Sciences 412 (1842) (1987) 53-74. arXiv:https://royalsocietypublishing.org/doi/pdf/10.1098/rspa.1987.0080, doi: 10.1098/rspa.1987.0080.

URL https://royalsocietypublishing.org/doi/abs/10.1098/rspa.1987.0080

\section{Appendix A. The 2D limit - freezing out $k_{z}$}

In this short appendix we will study for completeness why the momentum in $z$-direction can be expected to freeze out at $k_{z}=0$ if the sample is sufficiently thin. Since $v_{z}$ is the dominant energy scale as a first approximation one can neglect the terms $\propto v_{x, y}$. Therefore we may consider the Hamiltonian

$$
H=\hbar v_{z} \tau_{z} \otimes \sigma_{z} \hat{k}_{z}
$$

where $\sigma$ are Pauli matrices acting in orbital space and $\tau$ acts in spin space.

For a thin sample we expect electrons to stay localized in the sample and not leak out into the surroundings. Therefore we need a proper description of this and we shall choose the simplest description available for the Hamiltonian. One may recognize quite easily that a potential $V(z) \mathbb{1}_{4}$ for this type of Hamiltonian cannot localize particles in $z$-direction inside the sample. The simplest way to localize particles for this type of Hamiltonian is to add a mass-like term of form $M(z) \sigma_{x, y} \otimes \tau_{z, 0}$ or similar to the Hamiltonian [177-179]. Since this does not couple the different blocks of the Hamiltonian which are copies of each other up to a sign then it is sufficient to consider the different blocks separately. Therefore to understand how the electrons in our systems behave when trapped along the $z$-direction it is sufficient to consider the simpler problem of a Hamiltonian

$$
H=\hbar v_{z} \sigma_{z} \hat{k}_{z}+M(z) \sigma_{x, y}
$$

where

$$
M(z)= \begin{cases}\infty & z<-a / 2 \\ 0 & -a / 2<z<a / 2 \\ \infty & a / 2<z\end{cases}
$$


For $-a / 2<z<a / 2$ the Hamiltonian has eigenvectors $V_{1}=(1,0) e^{-i k z}$ and $V_{2}=(0,1) e^{i k z}$ that correspond to the same energy $E=\hbar v_{z} k$. Therefore a general solution at this energy is of the form $\psi=A V_{1}+B V_{2}$. It is easy to see [177-179] that possible boundary conditions at $z= \pm a / 2$ are

$$
\psi( \pm a / 2)=\sigma_{x, y} \psi( \pm a / 2)
$$

From here one can directly read off that $k$ has to fulfil

$$
e^{2 i a k}=1
$$

Therefore energy is quantized as

$$
E=\hbar v_{z} \pi \frac{n}{a} ; \quad n \in \mathbb{Z}
$$

Consequently, for sufficiently small $a$ the distance between energies is very large. Therefore at low energies $|E|<$ $\hbar v_{z} \pi / a$ one may set $k_{z}=0$ in the main text.

\section{Appendix B. Wavefunctions in the different spatial regions}

Appendix B.1. Wavefunctions for the isotropic $\left(k_{z} \neq 0\right)$ limit

To find the wavefunctons in the case of the massive rotation symmetric case we may now repeat the analysis from Sec. 3.1 for which some of the $k_{x, n}^{ \pm}$are physically allowed to find an ansatz for the wavefunction in region 1 . The ansatz is given by

$$
\begin{aligned}
& \Phi_{1}(x)=a_{1} e_{k, 1}^{+}\left(\begin{array}{c}
\tilde{\chi}_{k, 1}^{+(+)} \\
1 \\
0 \\
0
\end{array}\right)+a_{2} e_{k, 1}^{+}\left(\begin{array}{c}
0 \\
0 \\
\tilde{\chi}_{k, 1}^{+(-)} \\
1
\end{array}\right)+a_{3} e_{k, 2}^{+}\left(\begin{array}{c}
\tilde{\chi}_{k, 2}^{+(+)} \\
1 \\
0 \\
0
\end{array}\right)+a_{4} e_{k, 2}^{+}\left(\begin{array}{c}
0 \\
0 \\
\tilde{\chi}_{k, 2}^{+(-)} \\
1
\end{array}\right)+a_{5} e_{k, 1}^{-}\left(\begin{array}{c}
\tilde{\chi}_{k, 1}^{-(+)} \\
1 \\
0 \\
0
\end{array}\right)+a_{7} e_{k, 3}^{-}\left(\begin{array}{c}
\tilde{\chi}_{k, 3}^{-(+)} \\
1 \\
0 \\
0
\end{array}\right)+a_{8} e_{k, 3}^{-}\left(\begin{array}{c}
0 \\
0 \\
\tilde{\chi}_{k, 3}^{-(-)} \\
1
\end{array}\right), \\
& +a_{6} e_{k, 1}^{-}\left(\begin{array}{c}
0 \\
\tilde{\chi}_{k, 1}^{-(-)} \\
1
\end{array}\right)
\end{aligned}
$$

where we used the shorthand notation $e_{k, n}^{ \pm}=e^{i k_{x, n}^{ \pm} x}$ and defined

$$
\tilde{\chi}_{k, n}^{ \pm( \pm)}=\frac{\delta+( \pm) \sqrt{\left(k_{x, n}^{ \pm 2}+(k \sin \theta)^{2}\right)^{3}+\delta^{2}}}{\left(k_{x, n}^{ \pm}-i k \sin \theta\right)^{3}}
$$

In region 2 we have a modified wavevector amplitude $q=\left[(\epsilon-u)^{2}-\delta^{2}\right]^{1 / 6}$ and angle $\phi=\sin ^{-1}\left(\frac{k}{q} \sin \theta\right)$, which are found by enforcing energy and momentum conservation in $y$-direction. The $q_{x}$ component eigenvalues are like in the previous section given by Eq. (8) with $k \rightarrow q$ and $\theta \rightarrow \phi$. In this region, all of the $k_{n}^{ \pm}$are physically allowed, which dictates that the ansatz wavefunction in this region is given by

$$
\begin{aligned}
& \Phi_{2}(x)=b_{1} e_{q, 1}^{+}\left(\begin{array}{c}
\tilde{\chi}_{q, 1}^{+(-)} \\
1 \\
0 \\
0
\end{array}\right)+b_{2} e_{q, 1}^{+}\left(\begin{array}{c}
0 \\
0 \\
\tilde{\chi}_{q, 1}^{+(+)} \\
1
\end{array}\right)+b_{3} e_{q, 2}^{+}\left(\begin{array}{c}
\tilde{\chi}_{q, 2}^{+(-)} \\
1 \\
0 \\
0
\end{array}\right)+b_{4} e_{q, 2}^{+}\left(\begin{array}{c}
0 \\
0 \\
\tilde{\chi}_{q, 2}^{+(+)} \\
1
\end{array}\right)+b_{5} e_{q, 3}^{+}\left(\begin{array}{c}
\tilde{\chi}_{q, 3}^{+(-)} \\
1 \\
0 \\
0
\end{array}\right)+b_{6} e_{q, 3}^{+}\left(\begin{array}{c}
0 \\
0 \\
\tilde{\chi}_{q, 3}^{+(+)} \\
1
\end{array}\right) \\
& +b_{7} e_{q, 1}^{-}\left(\begin{array}{c}
\tilde{\chi}_{q, 1}^{-(-)} \\
1 \\
0 \\
0
\end{array}\right)+b_{8} e_{q, 1}^{-}\left(\begin{array}{c}
0 \\
0 \\
\tilde{\chi}_{q, 1}^{-(+)} \\
1
\end{array}\right)+b_{9} e_{q, 2}^{-}\left(\begin{array}{c}
\tilde{\chi}_{q, 2}^{-(-)} \\
1 \\
0 \\
0
\end{array}\right)+b_{10} e_{q, 2}^{-}\left(\begin{array}{c}
0 \\
0 \\
\tilde{\chi}_{q, 2}^{-(+)} \\
1
\end{array}\right)+b_{11} e_{q, 3}^{-}\left(\begin{array}{c}
\tilde{\chi}_{q, 3}^{-(-)} \\
1 \\
0 \\
0
\end{array}\right)+b_{12}^{-(-)} e_{q, 3}^{-}\left(\begin{array}{c}
0 \\
0 \\
\tilde{\chi}_{q, 3}^{-(+)} \\
1
\end{array}\right)\left(\begin{array}{c}
{ }^{-(+)} \\
0
\end{array}\right)
\end{aligned}
$$

where we made use of the shorthand notation (B.2). 
For region $3\left(x>\frac{L}{2}\right)$, where only propagation away from the barrier is allowed and terms need to be finite for $x \rightarrow+\infty$, we find that only $k_{x, 1}^{+}, k_{x, 2}^{-}$and $k_{x, 3}^{+}$fulfil these properties. Therefore, an ansatz for the spinor in this region is given by

$\Phi_{3}(x)=t_{1} e_{k, 1}^{+}\left(\begin{array}{c}\tilde{\chi}_{k, 1}^{+(+)} \\ 1 \\ 0 \\ 0\end{array}\right)+t_{2} e_{k, 1}^{+}\left(\begin{array}{c}0 \\ 0 \\ \tilde{\chi}_{k, 1}^{+(-)} \\ 1\end{array}\right)+t_{3} e_{k, 3}^{+}\left(\begin{array}{c}\tilde{\chi}_{k, 3}^{+(+)} \\ 1 \\ 0 \\ 0\end{array}\right)+t_{4} e_{k, 3}^{+}\left(\begin{array}{c}0 \\ 0 \\ \tilde{\chi}_{k, 3}^{+(-)} \\ 1\end{array}\right)+t_{5} e_{k, 2}^{-}\left(\begin{array}{c}\tilde{\chi}_{k, 2}^{-(+)} \\ 1 \\ 0 \\ 0\end{array}\right)+t_{6} e_{k, 2}^{-}\left(\begin{array}{c}0 \\ 0 \\ \tilde{\chi}_{k, 2}^{-(-)} \\ 1\end{array}\right)$.

Appendix B.2. Wavefunctions for the anisotropic $\left(k_{z}=0\right)$ limit

Much like previously also in the case of broken rotational symmetry we have to choose wavefunctions that correspond to wavevectors that are physically allowed. By doing so we find that the solution in region $\mathbf{1}$ is given as

$$
\begin{aligned}
& \Phi_{1}(x)=a_{1} e_{k, 1}^{+}\left(\begin{array}{c}
\chi_{k, 1}^{+} \\
1 \\
0 \\
0
\end{array}\right)+a_{2} e_{k, 1}^{+}\left(\begin{array}{c}
0 \\
0 \\
-\chi_{k, 1}^{+} \\
1
\end{array}\right)+a_{3} e_{k, 2}^{+}\left(\begin{array}{c}
\chi_{k, 2}^{+} \\
1 \\
0 \\
0
\end{array}\right)+a_{4} e_{k, 2}^{+}\left(\begin{array}{c}
0 \\
0 \\
-\chi_{k, 2}^{+} \\
1
\end{array}\right)+a_{5} e_{k, 1}^{-}\left(\begin{array}{c}
\chi_{k, 1}^{-} \\
1 \\
0 \\
0
\end{array}\right) \\
& +a_{6} e_{k, 1}^{-}\left(\begin{array}{c}
0 \\
0 \\
-\chi_{k, 1}^{-} \\
1
\end{array}\right)+a_{7} e_{k, 3}^{-}\left(\begin{array}{c}
\chi_{k, 3}^{-} \\
1 \\
0 \\
0
\end{array}\right)+a_{8} e_{k, 3}^{-}\left(\begin{array}{c}
0 \\
0 \\
-\chi_{k, 3}^{-} \\
1
\end{array}\right),
\end{aligned}
$$

where we have used the shorthand notation

$$
e_{k, n}^{ \pm}=e^{i k_{x, n}^{ \pm} x}
$$

and

$$
\chi_{k, n}^{ \pm}=\frac{\sqrt{\lambda^{2}\left(k_{x, n}^{ \pm}\right)^{2}\left(\left(k_{x, n}^{ \pm}\right)^{2}-3 k_{y}^{2}\right)^{2}+\left(k_{y}^{3}-3\left(k_{x, n}^{ \pm}\right)^{2} k_{y}\right)^{2}}}{\lambda\left(k_{x, n}^{ \pm}\right)^{3}-3 i\left(k_{x, n}^{ \pm}\right)^{2} k_{y}-3 \lambda\left(k_{x, n}^{ \pm}\right) k_{y}^{2}+i k_{y}^{3}} .
$$

with $k_{y}=k \sin \theta$.

Also in region $2\left(-\frac{L}{2}<x<\frac{L}{2}\right)$ one may use Eq. (28) to determine the allowed $q_{x}$ (renaming $k_{x} \rightarrow q_{x}$ ) for a given value of $k_{y}=k \sin \theta$. We may then use one of the $q_{x}$ (see more on this below) to define the wavevector amplitude $q=\sqrt{q_{x}^{2}+k_{y}^{2}}$ and angle $\phi=\arcsin \left(\frac{k \sin \theta}{q}\right)$. If we choose the $q_{x}$ solution (proper care is needed) one may then use Eq. (29) with the replacements $k \rightarrow q$ and $\theta \rightarrow \phi$ to have all the $q_{x}$ solutions expressed in a compact form. The wavefunction in this region is then given by

$$
\begin{aligned}
& \Phi_{2}(x)=b_{1} e_{q, 1}^{+}\left(\begin{array}{c}
-\chi_{q, 1}^{+} \\
1 \\
0 \\
0
\end{array}\right)+b_{2} e_{q, 1}^{+}\left(\begin{array}{c}
0 \\
0 \\
\chi_{q, 1}^{+} \\
1
\end{array}\right)+b_{3} e_{q, 2}^{+}\left(\begin{array}{c}
-\chi_{q, 2}^{+} \\
1 \\
0 \\
0
\end{array}\right)+b_{4} e_{q, 2}^{+}\left(\begin{array}{c}
0 \\
0 \\
\chi_{q, 2}^{+} \\
1
\end{array}\right)+b_{5} e_{q, 3}^{+}\left(\begin{array}{c}
-\chi_{q, 3}^{+} \\
1 \\
0 \\
0
\end{array}\right)+b_{6} e_{q, 3}^{+}\left(\begin{array}{c}
0 \\
0 \\
\chi_{q, 3}^{+} \\
1
\end{array}\right) \\
& +b_{7} e_{q, 1}^{-}\left(\begin{array}{c}
-\chi_{q, 1}^{-} \\
1 \\
0 \\
0
\end{array}\right)+b_{8} e_{q, 1}^{-}\left(\begin{array}{c}
0 \\
0 \\
\chi_{q, 1}^{-} \\
1
\end{array}\right)+b_{9} e_{q, 2}^{-}\left(\begin{array}{c}
-\chi_{q, 2}^{-} \\
1 \\
0 \\
0
\end{array}\right)+b_{10} e_{q, 2}^{-}\left(\begin{array}{c}
0 \\
0 \\
\chi_{q, 2}^{-} \\
1
\end{array}\right)+b_{11} e_{q, 3}^{-}\left(\begin{array}{c}
-\chi_{q, 3}^{-} \\
1 \\
0 \\
0
\end{array}\right)++b_{12} e_{q, 3}^{-}\left(\begin{array}{c}
0 \\
0 \\
\chi_{q, 3}^{-} \\
1
\end{array}\right),
\end{aligned}
$$

where we used the same shorthand notations as in region 1, Eqs. (B.6-B.7), just with replacements $k \rightarrow q$ and $\theta \rightarrow \phi$.

For region $3\left(x>\frac{L}{2}\right)$ we may use all definitions from region 1 without alteration and find a wavefunction ansatz as

$\Phi_{3}(x)=t_{1} e_{k, 1}^{+}\left(\begin{array}{c}\chi_{k, 1}^{+} \\ 1 \\ 0 \\ 0\end{array}\right)+t_{2} e_{k, 1}^{+}\left(\begin{array}{c}0 \\ 0 \\ -\chi_{k, 1}^{+} \\ 1\end{array}\right)+t_{3} e_{k, 3}^{+}\left(\begin{array}{c}\chi_{k, 3}^{+} \\ 1 \\ 0 \\ 0\end{array}\right)+t_{4} e_{k, 3}^{+}\left(\begin{array}{c}0 \\ 0 \\ -\chi_{k, 3}^{+} \\ 1\end{array}\right)+t_{5} e_{k, 2}^{-}\left(\begin{array}{c}\chi_{k, 2}^{-} \\ 1 \\ 0 \\ 0\end{array}\right)+t_{6} e_{k, 2}^{-}\left(\begin{array}{c}0 \\ 0 \\ -\chi_{k, 2}^{-} \\ 1\end{array}\right)$. 
Appendix B.3. Wavefunctions for the anisotropic $\left(k_{z} \neq 0\right)$ limit

For region $1\left(x<-\frac{L}{2}\right)$, we find that only some of the $k_{x, i}$ and their corresponding eigenvectors are allowed by the boundary conditions. That is the wavefunctions have to be finite as $x \rightarrow-\infty$. We find that the most general such wavefunction is given by

$$
\begin{aligned}
& \Phi_{1}(x)=a_{1} e_{k, 1}^{+}\left(\begin{array}{c}
\chi_{k, 1}^{+(+)} \\
1 \\
0 \\
0
\end{array}\right)+a_{2} e_{k, 1}^{+}\left(\begin{array}{c}
0 \\
0 \\
\chi_{k, 1}^{-(+)} \\
1
\end{array}\right)+a_{3} e_{k, 2}^{+}\left(\begin{array}{c}
\chi_{k, 2}^{+(+)} \\
1 \\
0 \\
0
\end{array}\right)+a_{4} e_{k, 2}^{+}\left(\begin{array}{c}
0 \\
0 \\
\chi_{k, 2}^{+(+)} \\
1
\end{array}\right)+a_{5} e_{k, 1}^{-}\left(\begin{array}{c}
\chi_{k, 1}^{+(-)} \\
1 \\
0 \\
0
\end{array}\right) \\
& +a_{6} e_{k, 1}^{-}\left(\begin{array}{c}
0 \\
0 \\
\chi_{k, 1}^{-(-)} \\
1
\end{array}\right)+a_{7} e_{k, 3}^{-}\left(\begin{array}{c}
\chi_{k, 3}^{+(-)} \\
1 \\
0 \\
0
\end{array}\right)+a_{8} e_{k, 3}^{-}\left(\begin{array}{c}
0 \\
0 \\
\chi_{k, 3}^{-(-)} \\
1
\end{array}\right)
\end{aligned}
$$

where we have used the shorthand notation

$$
e_{k, n}^{ \pm}=e^{i k_{x, n}^{ \pm} x}
$$

and

$$
\chi_{k, n}^{l \pm}=\frac{\delta+l \sqrt{\lambda^{2}\left(k_{x, n}^{ \pm}\right)^{2}\left[\left(k_{x, n}^{ \pm}\right)^{2}-3 k_{y}^{2}\right]^{2}+\left[k_{y}^{3}-3\left(k_{x, n}^{ \pm}\right)^{2} k_{y}\right]^{2}+\delta^{2}}}{\lambda\left(k_{x, n}^{ \pm}\right)^{3}-3 i\left(k_{x, n}^{ \pm}\right)^{2} k_{y}-3 \lambda\left(k_{x, n}^{ \pm}\right) k_{y}^{2}+i k_{y}^{3}}
$$

where $l= \pm 1$ and $k_{y}=k \sin \theta$.

In region $2\left(-\frac{L}{2}<x<\frac{L}{2}\right)$ we also have to determine possible wavevectors by solving Eq. (35) for $q_{x}$ (renaming $k_{x} \rightarrow q_{x}$ ). These solutions can also be written in the compact form (29) if we replace $k \rightarrow q$ and $\theta \rightarrow \phi$. Here we have to define $\phi=\arcsin \left(\frac{k \sin \theta}{q}\right)$ and $q=\sqrt{q_{x}^{2}+k_{y}^{2}}$. Of course proper care has to be taken to choose the proper real valued solution of $q_{x}$ that allows us to properly reproduce all solutions to (35). With these expressions in mind an ansatz for this region can be chosen to have the form

$$
\begin{aligned}
& \Phi_{2}(x)=b_{1} e_{q, 1}^{+}\left(\begin{array}{c}
\chi_{q, 1}^{-(+)} \\
1 \\
0 \\
0
\end{array}\right)+b_{2} e_{q, 1}^{+}\left(\begin{array}{c}
0 \\
0 \\
\chi_{q, 1}^{+(+)} \\
1
\end{array}\right)+b_{3} e_{q, 2}^{+}\left(\begin{array}{c}
\chi_{q, 2}^{-(+)} \\
1 \\
0 \\
0
\end{array}\right)+b_{4} e_{q, 2}^{+}\left(\begin{array}{c}
0 \\
0 \\
\chi_{q, 2}^{+(+)} \\
1
\end{array}\right)+b_{5} e_{q, 3}^{+}\left(\begin{array}{c}
\chi_{q, 3}^{-+} \\
1 \\
0 \\
0
\end{array}\right)+b_{6} e_{q, 3}^{+}\left(\begin{array}{c}
0 \\
0 \\
\chi_{q, 3}^{+(+)} \\
1
\end{array}\right) \\
& +b_{7} e_{q, 1}^{-}\left(\begin{array}{c}
\chi_{q, 1}^{-(-)} \\
1 \\
0 \\
0
\end{array}\right)+b_{8} e_{q, 1}^{-}\left(\begin{array}{c}
0 \\
0 \\
\chi_{q, 1}^{+(-)} \\
1
\end{array}\right)+b_{9} e_{q, 2}^{-}\left(\begin{array}{c}
\chi_{q, 2}^{-(-)} \\
1 \\
0 \\
0
\end{array}\right)+b_{10} e_{q, 2}^{-}\left(\begin{array}{c}
0 \\
0 \\
\chi_{q, 2}^{+(-)} \\
1
\end{array}\right)+b_{11} e_{q, 3}^{-}\left(\begin{array}{c}
\chi_{q, 3}^{--} \\
1 \\
0 \\
0
\end{array}\right)++b_{12} e_{q, 3}^{-}\left(\begin{array}{c}
0 \\
0 \\
\chi_{q, 3}^{+(-)} \\
1
\end{array}\right)
\end{aligned}
$$

Last, in region $3\left(x>\frac{L}{2}\right)$, taking into account that the wavefunction needs to be finite as $x \rightarrow+\infty$, we may therefore consider the following ansatz

$\Phi_{3}(x)=t_{1} e_{k, 1}^{+}\left(\begin{array}{c}\chi_{k, 1}^{+(+)} \\ 1 \\ 0 \\ 0\end{array}\right)+t_{2} e_{k, 1}^{+}\left(\begin{array}{c}0 \\ 0 \\ \chi_{k, 1}^{-(+)} \\ 1\end{array}\right)+t_{3} e_{k, 3}^{+}\left(\begin{array}{c}\chi_{k, 3}^{+(+)} \\ 1 \\ 0 \\ 0\end{array}\right)+t_{4} e_{k, 3}^{+}\left(\begin{array}{c}0 \\ 0 \\ \chi_{k, 3}^{-(+)} \\ 1\end{array}\right)+t_{5} e_{k, 2}^{-}\left(\begin{array}{c}\chi_{k, 2}^{+(-)} \\ 1 \\ 0 \\ 0\end{array}\right)+t_{6} e_{k, 2}^{-}\left(\begin{array}{c}0 \\ 0 \\ \chi_{k, 2}^{-(-)} \\ 1\end{array}\right)$

\section{Appendix C. Rotation for the tunneling Hamiltonian in z-direction}

In this appendix we discuss how the Hamiltonian can for the tunneling in the $z$-direction be brought into the form of equation (36). The Hamiltonian that one may consider for the more general case of a wave that has vector components $\left(k_{x}, k_{y}\right)=k \sin \theta(\cos \psi, \sin \psi)$ with an angle $\psi$ in the plane of the barrier is given as

$$
H(k)=\left(\begin{array}{cccc}
v_{z} / v k_{z} & \hat{k}_{1} & 0 & 0 \\
\hat{k}_{1}^{\dagger} & 0 & 0 & 0 \\
0 & 0 & 0 & -\hat{k}_{1} \\
0 & 0 & -\hat{k}_{1}^{\dagger} & 0
\end{array}\right)+u_{j} \mathbb{1}_{4},
$$


where $k_{1}=\frac{\lambda+1}{2} k_{+}^{3}+\frac{\lambda-1}{2} k_{-}^{3}$ as before. Through a unitary transformation $U^{\dagger} H(k) U$ one can bring the Hamiltonian into a form that only has momenta along the $z$ and $x$ directions. The transformation is given as

$$
U=\mathbb{1}_{2} \otimes e^{i / 2 \sigma_{3} \gamma} ; \quad \gamma=\arctan \left(\lambda^{-1} \tan (3 \psi)\right),
$$

where we used the usual notation adopted in the main text. The transformation corresponds to a rotation with angle $\gamma$ around the $z$-direction. With these changes we find equation (36) if we define the constant

$$
\nu=\frac{v_{z}}{v}\left(\lambda \cos (3 \psi) \sqrt{\frac{\tan ^{2}(3 \psi)}{\lambda^{2}}+1}\right)^{-1}
$$

and make use of a reduced energy that is defined slightly differently than in the rest of the text as indicated below

$$
\epsilon=\frac{E}{2 \hbar v}\left(\lambda \cos (3 \psi) \sqrt{\frac{\tan ^{2}(3 \psi)}{\lambda^{2}}+1}\right)^{-1} .
$$

